\title{
Initial boundary value problems for the two-component shallow water systems
}

\author{
Kai Yan and Zhaoyang Yin
}

\begin{abstract}
In this paper we study initial boundary value problems of three types of two-component shallow water systems on the half line subject to homogeneous Dirichlet boundary conditions. We first prove local wellpossedness of the two-component Camassa-Holm system, the modified two-component Camassa-Holm system, and the two-component DegasperisProcesi system in the Besov spaces. Then, we are able to specify certain conditions on the initial data which on the one hand guarantee global existence and on the other hand produce solutions with a finite lifespan. Moreover, in the case of finite time singularities we are able to describe the precise blow-up scenario for breaking waves. Finally we investigate global weak solutions for the two-component Camassa-Holm system and the modified two-component Camassa-Holm system on the half line, respectively. Our approach is based on sharp extension results for functions on the half line and several symmetry preserving properties of the systems under discussion.
\end{abstract}

\section{Introduction}

In this paper we present a thorough study on initial boundary value problems of three types of two-component shallow water systems on the half line.

The first type is the following integrable two-component Camassa-Holm shallow water system $(2 \mathrm{CH})$ :

$$
\begin{cases}m_{t}+u m_{x}+2 u_{x} m+\sigma \rho \rho_{x}=0, & t>0, x \in \mathbb{R}, \\ \rho_{t}+(u \rho)_{x}=0, & t>0, x \in \mathbb{R},\end{cases}
$$

where $m=u-u_{x x}$ and $\sigma= \pm 1$. The system (2CH) was recently derived by Constantin and Ivanov [16] in the context of shallow water theory. The variable $u(t, x)$

Mathematics Subject Classification (2010): 35G61, 35L05, 35L57.

Keywords: Two-component Camassa-Holm system, modified two-component Camassa-Holm system, two-component Degasperis-Procesi system, initial boundary value problems, local wellpossedness, Besov spaces, blow-up and global existence, global weak solutions. 
describes the horizontal velocity of the fluid and the variable $\rho(t, x)$ is connected with the horizontal deviation of the surface from equilibrium, all measured in dimensionless units [16]. The case $\sigma=1(\sigma=-1)$ corresponds to the situation in which the gravitational acceleration points downwards (upwards) [16]. The case $\sigma=1$ was originally proposed by Chen et al. in [7] and Falqui in [32], while $\sigma=-1$ is identified with the first negative flow of the AKNS hierarchy and has peakon and multikink solutions [7]. The extended $N=2$ supersymmetric Camassa-Holm equation was presented recently by Popowicz in [53].

For $\rho \equiv 0,(2 \mathrm{CH})$ becomes the Camassa-Holm equation $(\mathrm{CH})$, modeling the unidirectional propagation of shallow water waves over a flat bottom. Here $u(t, x)$ stands for the fluid velocity at time $t$ in the spatial $x$ direction, [5], [26], [45], [46]. $\mathrm{CH}$ is also a model for the propagation of axially symmetric waves in hyperelastic rods [22]. It has a bi-Hamiltonian structure [33] and is completely integrable (see [5] and [10]). There is also a geometric interpretation of $\mathrm{CH}$ in terms of the geodesic flow on the diffeomorphism group of the circle [18]. Its solitary waves are peaked [6]. They are orbitally stable and interact like solitons [2], [21]. It is also worth pointing out that the peaked solitary waves replicate a feature that is characteristic of waves of great height - waves of largest amplitude that are exact solutions of the governing equations for water waves, cf. the discussion in [11], [15] and [59].

The Cauchy problem and initial boundary value problem for $\mathrm{CH}$ have been studied extensively, see [12], [13], [23], [31] and [55]. It has been shown that this equation is locally well posed ([12], [13], [23], [55]) for initial data $u_{0} \in H^{s}(\mathbb{R})$, $s>3 / 2$. Moreover, it has global strong solutions ([9], [12], [13]) and also finite time blow-up solutions ([9], [12], [13], [14], [55]). On the other hand, it has global weak solutions in $H^{1}(\mathbb{R})$ ([3], [4], [20], [63]). The advantage of $\mathrm{CH}$ in comparison with the $\mathrm{KdV}$ equation lies in the fact that $\mathrm{CH}$ has peaked solitons and models wave breaking [6], [14] (by wave breaking we understand that the wave remains bounded while its slope becomes unbounded in finite time [62]).

For $\rho \not \equiv 0$, the Cauchy problems for $(2 \mathrm{CH})$ with $\sigma=-1$ and with $\sigma=1$ have been studied in [28] and [16], [35], [37], [39], [40], respectively. Local wellposedness for $(2 \mathrm{CH})$ with initial data in Sobolev spaces and in Besov spaces has been established in [16], [28], and [39]. The blow-up phenomena and global existence of strong solutions to $(2 \mathrm{CH})$ in Sobolev spaces have been derived in [28], [35], [39], and [40]. The analyticity of solutions to (2CH) has been obtained in [64]. Recently, the existence of global weak solutions for $(2 \mathrm{CH})$ with $\sigma=1$ has been investigated in [37].

Note that in the hydrodynamical derivation of $(2 \mathrm{CH})$ [16] it is required that $u(t, x) \rightarrow 0$ and $\rho(t, x) \rightarrow 1$ as $|x| \rightarrow \infty$, at any instant $t$. Then, setting $\eta \triangleq \rho-1$, we can rewrite the Cauchy problem of $(2 \mathrm{CH})$ with $\sigma=1$ as follows:

$$
\begin{cases}u_{t}-u_{t x x}+3 u u_{x}=2 u_{x} u_{x x}+u u_{x x x}-\eta \eta_{x}-\eta_{x}, & t>0, x \in \mathbb{R} \\ \eta_{t}+(u \eta)_{x}+u_{x}=0, & t>0, x \in \mathbb{R} \\ u(0, x)=u_{0}(x), & x \in \mathbb{R} \\ \eta(0, x)=\eta_{0}(x), & x \in \mathbb{R} .\end{cases}
$$


The second type of system considered here is the following modified twocomponent Camassa-Holm shallow water system (M2CH):

$$
\begin{cases}m_{t}+u m_{x}+2 u_{x} m+\sigma \rho \bar{\rho}_{x}=0, & t>0, x \in \mathbb{R} \\ \rho_{t}+(u \rho)_{x}=0, & t>0, x \in \mathbb{R}\end{cases}
$$

with $m=u-u_{x x}, \rho=\left(1-\partial_{x}^{2}\right)\left(\bar{\rho}-\bar{\rho}_{0}\right)$ and $\sigma= \pm 1$. The system (M2CH) was recently introduced by Holm et al. in [43]. It is written in terms of the velocity $u$ and the locally averaged density $\bar{\rho}$ (or depth, in the shallow-water interpretation) and $\overline{\rho_{0}}$ is taken to be constant. The system $(\mathrm{M} 2 \mathrm{CH})$ is defined as the geodesic motion on the semidirect product Lie group ([42], [52]) with respect to a certain metric and is given as a set of Euler-Poincaré equations on the dual of the corresponding Lie algebra.

For $\rho \equiv 0,(\mathrm{M} 2 \mathrm{CH})$ also becomes the classical Camassa-Holm equation. For $\rho \not \equiv 0$, many papers have been devoted to the Cauchy problem for $(\mathrm{M} 2 \mathrm{CH})$; see, for example, [36], [38], [56], [57], [58] and [64]. Local well-posedness and blowup phenomena for $(\mathrm{M} 2 \mathrm{CH})$ with initial data in Sobolev spaces have been studied in [36]. The analyticity of solutions to $(\mathrm{M} 2 \mathrm{CH})$ has been obtained in [64]. Recently, the existence of global weak solutions for $(\mathrm{M} 2 \mathrm{CH})$ has been investigated in [38], [56], [57], and [58].

For convenience, set $\gamma \triangleq \bar{\rho}-\bar{\rho}_{0}$. Then $\rho=\gamma-\gamma_{x x}$. We can rewrite the Cauchy problem for $(\mathrm{M} 2 \mathrm{CH})$ with $\sigma=1$ as follows:

$$
\begin{cases}m_{t}+u m_{x}+2 u_{x} m+\rho \gamma_{x}=0, & t>0, x \in \mathbb{R} \\ \rho_{t}+(u \rho)_{x}=0, & t>0, x \in \mathbb{R} \\ u(0, x)=u_{0}(x), & x \in \mathbb{R} \\ \gamma(0, x)=\gamma_{0}(x), & x \in \mathbb{R} .\end{cases}
$$

The third type of system considered here is the Cauchy problem for twocomponent Degasperis-Procesi system (2DP),

$$
\begin{cases}u_{t}-u_{t x x}+4 u u_{x}-3 u_{x} u_{x x}-u u_{x x x}+c \eta \eta_{x}=0, & t>0, x \in \mathbb{R} \\ \eta_{t}+u \eta_{x}+2 u_{x} \eta=0, & t>0, x \in \mathbb{R} \\ u(0, x)=u_{0}(x), & x \in \mathbb{R} \\ \eta(0, x)=\eta_{0}(x), & x \in \mathbb{R}\end{cases}
$$

where $c$ takes an arbitrary value. The system (1.3) as the Hamiltonian extension of the Degasperis-Procesi equation was first proposed in [54].

For $\eta \equiv 0$, system (1.3) becomes the classical Degasperis-Procesi equation (DP), see [25]. It was proved formally integrable by constructing a Lax pair [24], and the direct and inverse scattering approaches to studying it can be seen in [17]. Moreover, [24] also established that the DP equation has a bi-Hamiltonian structure and an infinite number of conservation laws, and admits exact peakon solutions which are analogous to the Camassa-Holm peakons. The DP equation is a model for nonlinear shallow water dynamics [19]. The numerical stability of solitons and peakons, the multi-soliton solutions and their peakon limits, together with 
an inverse scattering method to compute $n$-peakon solutions to the DP equation have been investigated respectively in [44], [50] and [51]. Furthermore, traveling wave solutions and the classification of all weak traveling wave solutions to the DP equation were presented in [47] and [61].

The Cauchy problem and the initial boundary value problem for the DP equation have been studied extensively, see [31], [41], and [66]. For example, the second author [66] established the local well-posedness of the DP equation with initial data $u_{0} \in H^{s}(\mathbb{R}), s>3 / 2$, on the line, and derived the precise blow-up scenario and a blow-up result. The global existence of strong solutions and global weak solutions for the DP equation were shown in [67] and [68]. Similar to the Camassa-Holm equation, the DP equation has not only global strong solutions [48], but also blowup solutions (see [29], [30], [48], and [67]). Apart from these, it has global entropy weak solutions in $L^{1}(\mathbb{R}) \cap B V(\mathbb{R})$ and $L^{2}(\mathbb{R}) \cap L^{4}(\mathbb{R})$, see [8].

Although DP is very similar to $\mathrm{CH}$ in many aspects, especially in the structure of the equation, there are some essential differences between the two equations. One of the famous features of the DP equation is that it has not only peakon solutions $u_{c}(t, x)=c e^{-|x-c t|}$ with $c>0$, [24], and periodic peakon solutions, [68], but also shock peakons [49] and periodic shock waves, [30]. Besides, $\mathrm{CH}$ is a reexpression of the geodesic flow on the diffeomorphism group [18], while the DP equation can be regarded as a nonmetric Euler equation [27].

For $\eta \not \equiv 0$, local well-posedness for system (1.3) in Besov spaces and blow-up phenomenon in Sobolev spaces have been researched in [65].

However, initial boundary value problems for the above three types of twocomponent systems on the half line have not been investigated so far. The aim of this paper is to find a general approach to investigate them. Our method strongly depends on sharp results on the odd extension of functions and the conservation of symmetry of the systems, by which we can convert initial boundary value problems on the half line into Cauchy problems on the whole line. Then, applying known results for Cauchy problems for the systems (1.1)-(1.3), we obtain local well-posedness results, blow-up and global existence results for strong solutions, and global weak solutions on the half line, which cover and improve the previous results for the classical $\mathrm{CH}$ and $\mathrm{DP}$ equations on the half line.

Our paper is organized as follows. In Section 2, we derive sharp extension results, which are crucial for our approach. In Section 3, by using the conservation of symmetry enjoyed by $(2 \mathrm{CH})$, we study initial boundary value problems of $(2 \mathrm{CH})$ on the half line. In Sections 4 and 5, we investigate initial boundary value problems of $(\mathrm{M} 2 \mathrm{CH})$ and $(2 \mathrm{DP})$ on the half line by the same method, respectively.

Notation. In the following, we denote by $*$ the spatial convolution. Given a Banach space $X$, we denote its norm by $\|\cdot\|_{X}$. If $s$ is a real number then we use the notation $s=[s]^{-}+\{s\}^{+}$, where $[s]^{-}$is an integer and $0<\{s\}^{+} \leq 1$.

Acknowledgments. The authors thank the referees for their valuable comments and suggestions. 


\section{Some crucial lemmas}

In this section, we present some key lemmas which will be crucial for our purposes. We here consider the case of the half line $(0, \infty)$.

Definition 2.1. Let $1<p<\infty$ and $-\infty<s<\infty$. Let $I=\mathbb{R}, I=\mathbb{R}_{+}$or let $I$ be an open interval of $\mathbb{R}$, where $\mathbb{R}_{+} \triangleq[0, \infty)$. The Besov space $B_{p, p}^{s}(I)$, the restriction of $B_{p, p}^{s}(\mathbb{R})(\mathrm{cf} .[60])$ to $I$, is defined as:

$$
B_{p, p}^{s}(I) \triangleq\left\{f \in \mathcal{D}^{\prime}(I): \exists g \in B_{p, p}^{s}(\mathbb{R}), \text { such that }\left.g\right|_{I}=f\right\},
$$

equipped with the norm

$$
\|f\|_{B_{p, p}^{s}(I)} \triangleq \inf _{g \in B_{p, p}^{s}(\mathbb{R}),\left.g\right|_{I}=f}\|g\|_{B_{p, p}^{s}(\mathbb{R})} .
$$

In particular, for $0<s$ not an integer, we have the following equivalent definition of $B_{p, p}^{s}(I)($ see $[1])$ :

$$
B_{p, p}^{s}(I) \triangleq\left\{u \in W^{m, p}(I):\|u\|_{B_{p, p}^{s}(I)}<\infty\right\}
$$

with

$$
\|u\|_{B_{p, p}^{s}(I)} \triangleq\|u\|_{W^{m, p}(I)}+\left(\int_{I} \int_{I} \frac{\left|u^{(m)}(x)-u^{(m)}(y)\right|^{p}}{|x-y|^{1+\sigma p}} d x d y\right)^{1 / p},
$$

where $s=m+\sigma, m \in \mathbb{N}$ and $\sigma \in(0,1)$.

Definition 2.2. Let $p, s$ and $I$ be as in the statement of Definition 2.1. The Banach space $X^{s, p}(I)$ is defined as follows:

$$
X^{s, p}(I) \triangleq \begin{cases}W^{s, p}(I), & \text { if } s \in \mathbb{N}, \\ B_{p, p}^{s}(I), & \text { if } 0<s \text { not an integer, or } s<0 .\end{cases}
$$

Lemma 2.3. Assume that $1<p<\infty$. We have:

(1) If $s \in[0,1 / p]$, then $C_{0}^{\infty}\left(\mathbb{R}_{+}\right)$is dense in $X^{s, p}\left(\mathbb{R}_{+}\right)$.

(2) If $s \in(1 / p, \infty)$, then $C_{0}^{\infty}\left(\mathbb{R}_{+}\right)$is dense in $X_{0}^{s, p}\left(\mathbb{R}_{+}\right)$, where

$$
X_{0}^{s, p}\left(\mathbb{R}_{+}\right) \triangleq\left\{f(x) \in W_{p}^{s}\left(\mathbb{R}_{+}\right): f^{(r)}(0)=0, r=0, \ldots,\left[s-\frac{1}{p}\right]^{-}\right\} .
$$

The proof of Lemma 2.3 is given on pages 219-220 of [60].

Lemma 2.4. Assume that $1<p<\infty$ and $0<s<1$ with $s \neq 1 / p$. If $f(x) \in$ $C_{0}^{\infty}\left(\mathbb{R}_{+}\right)$, then

$$
\int_{0}^{\infty} \frac{|f(x)|^{p}}{x^{p s}} d x \leq C(p, s) \int_{0}^{\infty} \int_{0}^{\infty} \frac{|f(x)-f(y)|^{p}}{|x-y|^{1+p s}} d x d y,
$$

where $C(p, s) \triangleq 2^{p-1}\left(1+(p-1)^{p-1}\left(\frac{2}{|1-p s|}\right)^{p}\right)$. 
Lemma 2.4 can be deduced from the conclusion on page 261 of [60] and Hardy's inequality, see page 262 of [60]. However, in order to obtain the exact constant in the above inequality and to show that $s=1 / p$ is a critical point for our problems, we give the complete proof.

Proof of Lemma 2.4. For $0<x<\infty$, we set

$$
g(x) \triangleq f(x)-\frac{1}{x} \int_{0}^{x} f(y) d y=\frac{1}{x} \int_{0}^{x}(f(x)-f(y)) d y .
$$

By $f(x) \in C_{0}^{\infty}\left(\mathbb{R}_{+}\right)$, we have

$$
\lim _{x \rightarrow 0} g(x)=\lim _{x \rightarrow \infty} g(x)=0 .
$$

Noting that

$$
\left(g(x)-\int_{x}^{\infty} \frac{g(y)}{y} d y-f(x)\right)^{\prime}=f^{\prime}(x)-\frac{f(x)}{x}+\frac{1}{x^{2}} \int_{0}^{x} f(y) d y+\frac{g(x)}{x}-f^{\prime}(x)=0,
$$

one can infer that

$$
f(x)=g(x)-\int_{x}^{\infty} \frac{g(y)}{y} d y
$$

Hence,

$$
|f(x)|^{p} \leq 2^{p-1}\left(|g(x)|^{p}+\left|\int_{x}^{\infty} \frac{g(y)}{y} d y\right|^{p}\right) .
$$

Case (1): $0<p s<1$. Thanks to Hölder's inequality, we have

$$
\begin{aligned}
\left|\int_{x}^{\infty} \frac{g(y)}{y} d y\right|^{p} & \leq\left(\int_{x}^{\infty} y^{\frac{1+p s-2 p}{2 p} q} d y\right)^{p / q} \cdot \int_{x}^{\infty}|g(y)|^{p} y^{-(1+p s) / 2} d y \\
& =\left(\frac{2 p}{(1-p s) q}\right)^{p / q} x^{(p s-1) / 2} \int_{x}^{\infty}|g(y)|^{p} y^{-(1+p s) / 2} d y
\end{aligned}
$$

where $1 / q=1-1 / p$.

The above inequality together with Fubini's theorem implies

$$
\begin{aligned}
\int_{0}^{\infty} x^{-p s} \mid & \left.\int_{x}^{\infty} \frac{g(y)}{y} d y\right|^{p} d x \\
& \leq\left(\frac{2 p}{(1-p s) q}\right)^{p / q} \int_{0}^{\infty} x^{-(1+p s) / 2}\left(\int_{x}^{\infty}|g(y)|^{p} y^{-(1+p s) / 2} d y\right) d x \\
& =\left(\frac{2 p}{(1-p s) q}\right)^{p / q} \int_{0}^{\infty}|g(y)|^{p} y^{-(1+p s) / 2}\left(\int_{0}^{y} x^{-(1+p s) / 2} d x\right) d y \\
& =\left(\frac{2 p}{(1-p s) q}\right)^{p / q} \frac{2}{1-p s} \int_{0}^{\infty}|g(y)|^{p} y^{-p s} d y \\
& =(p-1)^{p-1}\left(\frac{2}{1-p s}\right)^{p} \int_{0}^{\infty} \frac{|g(x)|^{p}}{x^{p s}} d x .
\end{aligned}
$$


Case (2): $p s>1$. In view of $(2.1)$, we have $\int_{0}^{\infty} g(y) / y d y=g(0)=0$. Thanks to Hölder's inequality, we deduce

$$
\begin{aligned}
\left|\int_{x}^{\infty} \frac{g(y)}{y} d y\right|^{p} & =\left|\int_{0}^{x} \frac{g(y)}{y} d y\right|^{p} \leq\left(\int_{0}^{x} y^{\frac{1+p s-2 p}{2 p} q} d y\right)^{p / q} \int_{0}^{x}|g(y)|^{p} y^{-(1+p s) / 2} d y \\
& =\left(\frac{2 p}{(p s-1) q}\right)^{p / q} x^{(p s-1) / 2} \int_{0}^{x}|g(y)|^{p} y^{-(1+p s) / 2} d y,
\end{aligned}
$$

where $1 / q=1-1 / p$.

By using Fubini's theorem again, we obtain

$$
\begin{aligned}
\int_{0}^{\infty} x^{-p s} \mid & \left.\int_{x}^{\infty} \frac{g(y)}{y} d y\right|^{p} d x \\
\leq & \left(\frac{2 p}{(p s-1) q}\right)^{p / q} \int_{0}^{\infty} x^{-(1+p s) / 2}\left(\int_{0}^{x}|g(y)|^{p} y^{-(1+p s) / 2} d y\right) d x \\
& =\left(\frac{2 p}{(p s-1) q}\right)^{p / q} \int_{0}^{\infty}|g(y)|^{p} y^{-(1+p s) / 2}\left(\int_{y}^{\infty} x^{-(1+p s) / 2} d x\right) d y \\
& =(p-1)^{p-1}\left(\frac{2}{p s-1}\right)^{p} \int_{0}^{\infty} \frac{|g(x)|^{p}}{x^{p s}} d x .
\end{aligned}
$$

Consequently, for $s \in(0,1)$ with $s \neq 1 / p$, we get

$$
\int_{0}^{\infty} x^{-p s}\left|\int_{x}^{\infty} \frac{g(y)}{y} d y\right|^{p} d x \leq(p-1)^{p-1}\left(\frac{2}{|p s-1|}\right)^{p} \int_{0}^{\infty} \frac{|g(x)|^{p}}{x^{p s}} d x .
$$

Combining (2.2) and (2.3), and taking advantage of Hölder's inequality and Fubini's theorem again, we obtain

$$
\begin{aligned}
\int_{0}^{\infty} \frac{|f(x)|^{p}}{x^{p s}} d x & \leq 2^{p-1} \int_{0}^{\infty} \frac{|g(x)|^{p}}{x^{p s}} d x+2^{p-1} \int_{0}^{\infty} x^{-p s}\left|\int_{x}^{\infty} \frac{g(y)}{y} d y\right|^{p} d x \\
& \leq 2^{p-1}\left(1+(p-1)^{p-1}\left(\frac{2}{|1-p s|}\right)^{p}\right) \int_{0}^{\infty} \frac{|g(x)|^{p}}{x^{p s}} d x \\
& \leq C(p, s) \int_{0}^{\infty} x^{-p s-1}\left(\int_{0}^{x}|f(x)-f(y)|^{p} d y\right) d x \\
& =C(p, s) \int_{0}^{\infty} \int_{y}^{\infty} x^{-p s-1}|f(x)-f(y)|^{p} d x d y \\
& =C(p, s) \int_{0}^{\infty} \int_{0}^{\infty}(x+y)^{-p s-1}|f(x+y)-f(y)|^{p} d x d y \\
& \leq C(p, s) \int_{0}^{\infty} \int_{0}^{\infty} x^{-p s-1}|f(x+y)-f(y)|^{p} d x d y \\
& \leq C(p, s) \int_{0}^{\infty} \int_{0}^{\infty} \frac{|f(x)-f(y)|^{p}}{|x-y|^{1+p s}} d x d y .
\end{aligned}
$$

This completes the proof of the lemma. 
Lemma 2.5. Let $1<p<\infty, 0 \leq s \leq 1$ with $s \neq 1 / p$. Assume that $v \in X^{s, p}\left(\mathbb{R}_{+}\right)$ if $0 \leq s<1 / p$ or that $v \in X^{s, p}\left(\mathbb{R}_{+}\right)$with $v(0)=0$ if $1 / p<s \leq 1$. Furthermore let

$$
\tilde{v}(x)= \begin{cases}v(x), & \text { if } x \geq 0 \\ -v(-x), & \text { if } x<0\end{cases}
$$

Then $\tilde{v}(x) \in X^{s, p}(\mathbb{R})$.

Proof. It is obvious that the lemma holds true for $s=0$.

For $s=1$, we have $v(x) \in W_{0}^{1, p}\left(\mathbb{R}_{+}\right)$. If $v \in C^{1}\left(\mathbb{R}_{+}\right) \cap W_{0}^{1, p}\left(\mathbb{R}_{+}\right)$, then one can readily obtain that

$$
\lim _{x \rightarrow 0^{-}} \frac{\tilde{v}(x)-\tilde{v}(0)}{x-0}=\lim _{x \rightarrow 0^{-}} \frac{-v(-x)}{x}=\lim _{x \rightarrow 0^{+}} \frac{v(x)}{x}=v^{\prime}\left(0^{+}\right)=\lim _{x \rightarrow 0^{+}} \frac{\tilde{v}(x)-\tilde{v}(0)}{x-0},
$$

which implies $\tilde{v} \in C^{1}(\mathbb{R}) \cap W^{1, p}(\mathbb{R})$. Since $C^{1}\left(\mathbb{R}_{+}\right) \cap W_{0}^{1, p}\left(\mathbb{R}_{+}\right)$is dense in $W_{0}^{1, p}\left(\mathbb{R}_{+}\right)$, it follows that the lemma is true for $s=1$.

Next, we prove that the lemma is true for $0<s<1$, with $s \neq 1 / p$. For $v \in C_{0}^{\infty}\left(\mathbb{R}_{+}\right)$, by the definition of $\tilde{v}$, Lemma 2.4 and the fact that $|v(x)+v(y)|^{p} \leq$ $2^{p-1}\left(|v(x)|^{p}+|v(y)|^{p}\right)$, we have

$$
\begin{array}{rl}
\int_{\mathbb{R}} \int_{\mathbb{R}} \frac{|\tilde{v}(x)-\tilde{v}(y)|^{p}}{\mid x}-\left.y\right|^{1+p s} & d x d y \\
& =2 \int_{0}^{\infty} \int_{0}^{\infty} \frac{|v(x)-v(y)|^{p}}{|x-y|^{1+p s}} d x d y+2 \int_{0}^{\infty} \int_{0}^{\infty} \frac{|v(x)+v(y)|^{p}}{|x+y|^{1+p s}} d x d y \\
& \leq 2 \int_{0}^{\infty} \int_{0}^{\infty} \frac{|v(x)-v(y)|^{p}}{|x-y|^{1+p s}} d x d y+2^{p} \int_{0}^{\infty} \int_{0}^{\infty} \frac{|v(x)|^{p}+|v(y)|^{p}}{|x+y|^{1+p s}} d x d y \\
& =2 \int_{0}^{\infty} \int_{0}^{\infty} \frac{|v(x)-v(y)|^{p}}{|x-y|^{1+p s}} d x d y+\frac{2^{p+1}}{p s} \int_{0}^{\infty} \frac{|v(x)|^{p}}{|x|^{p s}} d x d y \\
& \leq\left(2+\frac{2^{p+1}}{p s} C(p, s)\right) \int_{0}^{\infty} \int_{0}^{\infty} \frac{|v(x)-v(y)|^{p}}{|x-y|^{1+p s}} d x d y,
\end{array}
$$

which together with Definition 2.1 and 2.2 yields $\tilde{v} \in W^{p, s}(\mathbb{R})$, provided that $v \in C_{0}^{\infty}\left(\mathbb{R}_{+}\right)$. In view of Lemma 2.3 and the assumption of this lemma, one can get the desired result. Therefore, we the proof of the lemma is complete.

Remark 2.6. For the limit case $p=\infty$, we have

(1) if $v \in L^{\infty}\left(\mathbb{R}_{+}\right)$, then $\tilde{v} \in L^{\infty}(\mathbb{R})$.

(2) if $v \in W^{1, \infty}\left(\mathbb{R}_{+}\right)$with $v(0)=0$, then $\tilde{v} \in W^{1, \infty}(\mathbb{R})$.

Noting that $C^{1}\left(\mathbb{R}_{+}\right) \cap W^{1, \infty}\left(\mathbb{R}_{+}\right)$is not dense in $W^{1, \infty}\left(\mathbb{R}_{+}\right)$, we cannot prove Remark 2.6(2) in the same way as in the proof of Lemma 2.5. To this end, we first need the following proposition: 
Proposition 2.7. Assume that $g \in L^{\infty}(\mathbb{R})$. If we set

$$
h(x) \triangleq \int_{0}^{x} g(t) d t, \quad \forall x \in \mathbb{R},
$$

then $h(x) \in C(\mathbb{R})$ and

$$
\int_{\mathbb{R}} h(x) \varphi^{\prime}(x) d x=-\int_{\mathbb{R}} g(x) \varphi(x) d x, \quad \forall \varphi \in C_{c}^{1}(\mathbb{R}) .
$$

That is, the weak derivative of $h$, denoted by $h^{\prime}$, is $g$.

Proof. It is obvious that $h(x) \in C(\mathbb{R})$.

Next, we prove that $h^{\prime}=g$. By Fubini's theorem, we have, for $\varphi \in C_{c}^{1}(\mathbb{R})$,

$$
\begin{aligned}
\int_{\mathbb{R}} h(x) \varphi^{\prime}(x) d x & =\int_{\mathbb{R}}\left(\int_{0}^{x} g(t) d t\right) \varphi^{\prime}(x) d x \\
& =-\int_{-\infty}^{0}\left(\int_{x}^{0} g(t) d t\right) \varphi^{\prime}(x) d x+\int_{0}^{\infty}\left(\int_{0}^{x} g(t), d t\right) \varphi^{\prime}(x) d x \\
& =-\int_{-\infty}^{0} g(t)\left(\int_{-\infty}^{t} \varphi^{\prime}(x) d x\right) d t+\int_{0}^{\infty} g(t)\left(\int_{t}^{\infty} \varphi^{\prime}(x) d x\right) d t \\
& =-\int_{-\infty}^{0} g(t) \varphi(t) d t-\int_{0}^{\infty} g(t) \varphi(t) d t \\
& =-\int_{\mathbb{R}} g(x) \varphi(x) d x,
\end{aligned}
$$

which implies the desired result.

Proof of Remark 2.6. It is obvious that (1) is true. For (2), by the assumption $v \in W^{1, \infty}\left(\mathbb{R}_{+}\right)$, we have $\tilde{v} \in L^{\infty}(\mathbb{R})$. Now it suffices to show that $\tilde{v}$ is weakly differentiable and $\tilde{v}^{\prime} \in L^{\infty}(\mathbb{R})$. Indeed, set

$$
v^{\star}(x) \triangleq \begin{cases}v^{\prime}(x), & \text { if } x>0, \\ v^{\prime}(-x), & \text { if } x<0 .\end{cases}
$$

Hence, $v^{\star}(x) \in L^{\infty}(\mathbb{R})$. Noting that $\tilde{v}(0)=v(0)=0$ and using the definition of $v^{\star}$, we can readily get

$$
\tilde{v}(x)=\int_{0}^{x} v^{\star}(t) d t, \quad \forall x \in \mathbb{R} .
$$

Then Proposition 2.7 implies $\tilde{v}^{\prime}=v^{\star} \in L^{\infty}(\mathbb{R})$. Therefore, the proof of the remark is complete.

Lemma 2.8. Let $1<p<\infty$ and $0 \leq s \leq 1$. Assume that $v \in X^{s, p}\left(\mathbb{R}_{+}\right)$. Furthermore, let

$$
\hat{v}(x)= \begin{cases}v(x), & \text { if } x \geq 0 \\ v(-x), & \text { if } x<0\end{cases}
$$

Then $\hat{v}(x) \in X^{s, p}(\mathbb{R})$. 
Proof. It is obvious that the lemma is true for $s=0$.

For $s=1$, we have $v \in W^{1, p}\left(\mathbb{R}_{+}\right)$. If $v \in C^{1}\left(\mathbb{R}_{+}\right) \cap W^{1, p}\left(\mathbb{R}_{+}\right)$, then we get $v \in C_{\text {loc }}^{0,1}\left(\mathbb{R}_{+}\right)$. Hence, $\hat{v} \in C_{\text {loc }}^{0,1}(\mathbb{R})$. Note that a locally uniformly Lipschitz continuous function is weakly differentiable, cf. [34]. We have

$$
(\hat{v})^{\prime}(x)=\widetilde{v^{\prime}}(x)= \begin{cases}v^{\prime}(x), & \text { if } x \geq 0, \\ -v^{\prime}(-x), & \text { if } x<0 .\end{cases}
$$

A straightforward calculation yields

$$
\|\hat{v}(x)\|_{W^{1, p}(\mathbb{R})}^{p}=2\|v(x)\|_{W^{1, p}\left(\mathbb{R}_{+}\right)}^{p} .
$$

Noting that $C^{1}\left(\mathbb{R}_{+}\right) \cap W^{1, p}\left(\mathbb{R}_{+}\right)$is dense in $W^{1, p}\left(\mathbb{R}_{+}\right)$, one can infer that $\hat{v}(x) \in$ $W^{1, p}(\mathbb{R})$.

Next, we prove that the lemma holds true for $0<s<1$. By the definition of $\hat{v}$ and the fact $|x+y| \geq|x-y|$ for $x, y \geq 0$, one deduce that

$$
\begin{aligned}
& \int_{\mathbb{R}} \int_{\mathbb{R}} \frac{|\hat{v}(x)-\hat{v}(y)|^{p}}{|x-y|^{1+p s}} d x d y \\
& \quad=2 \int_{0}^{\infty} \int_{0}^{\infty} \frac{|v(x)-v(y)|^{p}}{|x-y|^{1+p s}} d x d y+2 \int_{0}^{\infty} \int_{0}^{\infty} \frac{|v(x)-v(y)|^{p}}{|x+y|^{1+p s}} d x d y \\
& \leq 4 \int_{0}^{\infty} \int_{0}^{\infty} \frac{|v(x)-v(y)|^{p}}{|x-y|^{1+p s}} d x d y
\end{aligned}
$$

By Lemma 2.3, we obtain the desired result. This completes the proof of the lemma.

Remark 2.9. For the limit case $p=\infty$, in view of the definition of $\hat{v}$, one can easily get $\hat{v} \in L^{\infty}(\mathbb{R})$ or $\hat{v} \in W^{1, \infty}(\mathbb{R})$ provided that $v \in L^{\infty}\left(\mathbb{R}_{+}\right)$or $v \in W^{1, \infty}\left(\mathbb{R}_{+}\right)$.

Lemma 2.10. Assume that $1<p<\infty$ and $\sigma \in[0,1]$. If $u \in W^{m, p}(I)$, with $m \in \mathbb{N}_{+}$, then $u \in X^{m+\sigma, p}(I)$ if and only if $u^{(m)} \in X^{\sigma, p}(I)$.

Proof. This is a direct consequence of Definitions 2.1 and 2.2.

Lemma 2.11. Let $1<p<\infty$ and $1 / p<s<2+1 / p$. Assume that $v \in X^{s, p}\left(\mathbb{R}_{+}\right)$ with $v(0)=0$. Furthermore, let

$$
\tilde{v}(x)= \begin{cases}v(x), & \text { if } x \geq 0, \\ -v(-x), & \text { if } x<0 .\end{cases}
$$

Then $\tilde{v}(x) \in X^{s, p}(\mathbb{R})$.

Proof. For $1 / p<s \leq 1$, the lemma is a consequence of Lemma 2.5.

For $1 \leq s \leq 2$, we have $s-1 \in[0,1]$ and

$$
\tilde{v}^{\prime}(x)=\widehat{v^{\prime}}(x)= \begin{cases}v^{\prime}(x), & \text { if } x \geq 0 \\ v^{\prime}(-x), & \text { if } x<0 .\end{cases}
$$


In view of Lemma 2.10, one has $v^{\prime}(x) \in X^{s-1, p}\left(\mathbb{R}_{+}\right)$. It follows from Lemma 2.8 that $\tilde{v}^{\prime}(x)=\widehat{v^{\prime}}(x) \in X^{s-1, p}(\mathbb{R})$. Noting that $\tilde{v}(x) \in W^{1, p}(\mathbb{R})$ and using Lemma 2.10 again, we have $\tilde{v}(x) \in X^{s, p}(\mathbb{R})$ for $1 \leq s \leq 2$.

For $2 \leq s<2+1 / p$, we have $s-2 \in[0,1 / p)$ and

$$
\tilde{v}^{\prime \prime}(x)=\widetilde{v^{\prime \prime}}(x)= \begin{cases}v^{\prime \prime}(x), & \text { if } x \geq 0, \\ -v^{\prime \prime}(-x), & \text { if } x<0 .\end{cases}
$$

In view of Lemma 2.10, one has $v^{\prime \prime}(x) \in X^{s-2, p}\left(\mathbb{R}_{+}\right)$. It follows from Lemma 2.5 that $\tilde{v}^{\prime \prime}(x)=\widetilde{v^{\prime \prime}}(x) \in X^{s-2, p}(\mathbb{R})$. Noting that $\tilde{v}(x) \in W^{2, p}(\mathbb{R})$ and using Lemma 2.10 again, we have $\tilde{v}(x) \in X^{s, p}(\mathbb{R})$ for $2 \leq s<2+1 / p$. This completes the proof of the lemma.

Remark 2.12. For $s \geq 2+1 / p$, under the assumptions of Lemma 2.11, generally one cannot deduce $\tilde{v} \in X^{s, p}(\mathbb{R})$.

In order to obtain $\tilde{v} \in X^{s, p}(\mathbb{R})$, one has to add additional conditions. For this we let $k \in \mathbb{N}$, and for $2 k+1 / p<s<2 k+2+1 / p$ we set

$$
Y_{k}^{s, p}\left(\mathbb{R}_{+}\right)=\left\{v \in X^{s, p}\left(\mathbb{R}_{+}\right): v^{(2 k)}(0)=v^{(2 k-2)}(0)=\cdots=v(0)=0\right\} .
$$

We now have the following generalized result:

Lemma 2.13. For $k \in \mathbb{N}$ and $2 k+1 / p<s<2 k+2+1 / p$, assume that $v \in$ $Y_{k}^{s, p}\left(\mathbb{R}_{+}\right)$. Furthermore, let

$$
\tilde{v}(x)= \begin{cases}v(x), & \text { if } x \geq 0, \\ -v(-x), & \text { if } x<0 .\end{cases}
$$

Then $\tilde{v}(x) \in X^{s, p}(\mathbb{R})$.

Proof. By following the lines of the proof of Lemma 2.11, one can easily prove the lemma by induction with respect to the index $k$. For the sake of brevity, we omit the details here.

Lemma 2.14. Let $1<p<\infty$ and $0 \leq s<1+1 / p$. Assume that $v \in X^{s, p}\left(\mathbb{R}_{+}\right)$. Furthermore, let

$$
\hat{v}(x)= \begin{cases}v(x), & \text { if } x \geq 0 \\ v(-x), & \text { if } x<0\end{cases}
$$

Then $\hat{v}(x) \in X^{s, p}(\mathbb{R})$.

Proof. For $s \in[0,1]$, the lemma is a direct consequence of Lemma 2.8.

For $1<s<1+1 / p$, we have $v \in B_{p, p}^{1+\sigma}\left(\mathbb{R}_{+}\right)$with $\sigma \in(0,1 / p)$. Thus, $v \in$ $W^{1, p}\left(\mathbb{R}_{+}\right)$. Thanks to Lemma 2.8 , we have $\hat{v} \in W^{1, p}(\mathbb{R})$ and

$$
\hat{v}^{\prime}(x)=\widetilde{v^{\prime}}(x)= \begin{cases}v^{\prime}(x), & \text { if } x \geq 0, \\ -v^{\prime}(-x), & \text { if } x<0 .\end{cases}
$$

By Lemma 2.10, we only need to show that $\hat{v}^{\prime}(x) \in B_{p, p}^{\sigma}(\mathbb{R})$ with $\sigma \in(0,1 / p)$. Indeed, this can be deduced from the fact $v^{\prime}(x) \in B_{p, p}^{\sigma}\left(\mathbb{R}_{+}\right)$and Lemma 2.5. Therefore, the proof of the lemma is complete. 
Remark 2.15. For $s \geq 1+1 / p$, under the assumptions of Lemma 2.14, generally one cannot deduce $\hat{v} \in X^{s, p}(\mathbb{R})$.

In order to obtain $\hat{v} \in X^{s, p}(\mathbb{R})$, one has to add additional conditions. For this we let $k \in \mathbb{N}_{+}$, and for $2 k-1+1 / p<s<2 k+1+1 / p$ we set

$$
Z_{k}^{s, p}\left(\mathbb{R}_{+}\right)=\left\{v \in X^{s, p}\left(\mathbb{R}_{+}\right): v^{(2 k-1)}(0)=v^{(2 k-3)}(0)=\cdots=v^{\prime}(0)=0\right\} .
$$

We now have the following generalized lemma:

Lemma 2.16. For $k \in \mathbb{N}_{+}$and $2 k-1+1 / p<s<2 k+1+1 / p$, assume that $v \in Z_{k}^{s, p}\left(\mathbb{R}_{+}\right)$. Furthermore, let

$$
\hat{v}(x)= \begin{cases}v(x), & \text { if } x \geq 0 \\ v(-x), & \text { if } x<0\end{cases}
$$

Then $\hat{v}(x) \in X^{s, p}(\mathbb{R})$.

Proof. By following the lines of the proof of Lemma 2.14, one can easily prove the lemma by induction with respect to the index $k$. We omit the details.

For later applications of the above lemmas, we need the following new spaces:

Definition 2.17. (1) Let $1<p<\infty,-\infty<s<\infty, T>0$ and $I=\mathbb{R}$ or $I=\mathbb{R}_{+}$, where $\mathbb{R}_{+} \triangleq[0, \infty)$. The spaces $V^{s, p}(I)$ and $E_{T}^{s, p}(I)$ are defined as follows:

$$
\begin{gathered}
V^{s, p}(I) \triangleq \begin{cases}H^{s}(I), & \text { if } s \in \mathbb{N}, \\
B_{p, p}^{s}(I), & \text { if } 0<s \text { not an integer, or } s<0,\end{cases} \\
E_{T}^{s, p}(I) \triangleq C\left([0, T) ; V^{s, p}(I)\right) \cap C^{1}\left([0, T) ; V^{s-1, p}(I)\right) .
\end{gathered}
$$

In particular, $V^{s, 2}(I)=H^{s}(I)$ and

$$
E_{T}^{s, 2}(I) \triangleq C\left([0, T) ; H^{s}(I)\right) \cap C^{1}\left([0, T) ; H^{s-1}(I)\right) .
$$

(2) For $k \in \mathbb{N}$ and $2 k+1 / p<s<2 k+2+1 / p$, we set

$$
D_{k}^{s, p}\left(\mathbb{R}_{+}\right) \triangleq\left\{v \in V^{s, p}\left(\mathbb{R}_{+}\right): v^{(2 k)}(0)=v^{(2 k-2)}(0)=\cdots=v(0)=0\right\} .
$$

(3) For $k \in \mathbb{N}_{+}$and $2 k-1+1 / p<s<2 k+1+1 / p$, we set

$$
R_{k}^{s, p}\left(\mathbb{R}_{+}\right) \triangleq\left\{v \in V^{s, p}\left(\mathbb{R}_{+}\right): v^{(2 k-1)}(0)=v^{(2 k-3)}(0)=\cdots=v^{\prime}(0)=0\right\} .
$$

Remark 2.18. Obviously, the extension of Lemmas 2.5, 2.8, 2.11, and 2.14 also hold true for the space $V^{s, p}$, a special case of $X^{s, p}$. And the generalized extensions of Lemmas 2.13 and 2.16 can be also applied to the spaces $D_{k}^{s, p}$ and $R_{k}^{s, p}$, respectively. 


\section{IBVP for two-component Camassa-Holm shallow water system}

In this section, we investigate initial boundary value problems of the two-component Camassa-Holm shallow water system on the half line.

Let us now consider the following initial boundary value problem for $(2 \mathrm{CH})$ on the half line:

$$
\begin{cases}u_{t}-u_{t x x}+3 u u_{x}=2 u_{x} u_{x x}+u u_{x x x}-\eta \eta_{x}-\eta_{x}, & t>0, x \in \mathbb{R}_{+}, \\ \eta_{t}+(u \eta)_{x}+u_{x}=0, & t>0, x \in \mathbb{R}_{+}, \\ u(0, x)=u_{0}(x), & x \in \mathbb{R}_{+}, \\ \eta(0, x)=\eta_{0}(x), & x \in \mathbb{R}_{+} \\ u(t, 0)=0, & t \geq 0\end{cases}
$$

We first present the following local well-posedness result for the system (3.1).

Theorem 3.1. Let $1<p<\infty$ and $\max (3 / 2,1+1 / p, 2-1 / p)<s<2+1 / p$. Assume that $\left(u_{0}, \eta_{0}\right) \in V^{s, p}\left(\mathbb{R}_{+}\right) \times V^{s-1, p}\left(\mathbb{R}_{+}\right)$satisfies the compatibility condition $u_{0}(0)=0$. Then there exists a maximal $T=T\left(u_{0}, \eta_{0}\right)>0$ such that the system (3.1) has a unique solution $(u(t, x), \eta(t, x)) \in E_{T}^{s, p}\left(\mathbb{R}_{+}\right) \times E_{T}^{s-1, p}\left(\mathbb{R}_{+}\right)$. Moreover, the solution depends continuously on the initial data, i.e., the mapping

$$
\left(u_{0}, \eta_{0}\right) \mapsto(u, \eta): V^{s, p}\left(\mathbb{R}_{+}\right) \times V^{s-1, p}\left(\mathbb{R}_{+}\right) \rightarrow E_{T}^{s, p}\left(\mathbb{R}_{+}\right) \times E_{T}^{s-1, p}\left(\mathbb{R}_{+}\right)
$$

is continuous. Furthermore, the maximal $T$ is independent of $s$ in the following sense: if $(u, \eta) \in E_{T}^{s, 2}\left(\mathbb{R}_{+}\right) \times E_{T}^{s-1,2}\left(\mathbb{R}_{+}\right)$is the solution to the system (3.1), and if $\left(u_{0}, \eta_{0}\right) \in H^{s^{\prime}}\left(\mathbb{R}_{+}\right) \times H^{s^{\prime}-1}\left(\mathbb{R}_{+}\right)$with $3 / 2<s \leq s^{\prime}<5 / 2$ satisfies $u_{0}(0)=0$, then $(u, \eta) \in E_{T}^{s^{\prime}, 2}\left(\mathbb{R}_{+}\right) \times E_{T}^{s^{\prime}-1,2}\left(\mathbb{R}_{+}\right)$with the same $T$.

Proof. We first convert (3.1) into the Cauchy problem for $(2 \mathrm{CH})$ on the line. For this, we extend the initial data $u_{0}(x)$ and $\eta_{0}(x)$ defined on the half line into the odd and even functions on the line defined, respectively, by

$$
\tilde{u}_{0}(x)= \begin{cases}u_{0}(x), & \text { if } x \geq 0 \\ -u_{0}(-x), & \text { if } x<0\end{cases}
$$

and

$$
\hat{\eta}_{0}(x)= \begin{cases}\eta_{0}(x), & \text { if } x \geq 0 \\ \eta_{0}(-x), & \text { if } x<0 .\end{cases}
$$

By the assumption of the theorem, in view of Lemmas 2.11 and Lemma 2.14, one obtain

$$
\left(\tilde{u}_{0}(x), \hat{\eta}_{0}(x)\right) \in V^{s, p}(\mathbb{R}) \times V^{s-1, p}(\mathbb{R})
$$

with $\max (3 / 2,1+1 / p, 2-1 / p)<s<2+1 / p$.

We now can convert (3.1) into the following system on the line:

$$
\begin{cases}\tilde{u}_{t}-\tilde{u}_{t x x}+3 \tilde{u} \tilde{u}_{x}=2 \tilde{u}_{x} \tilde{u}_{x x}+\tilde{u} \tilde{u}_{x x x}-\hat{\eta} \hat{\eta}_{x}-\hat{\eta}_{x}, & t>0, x \in \mathbb{R}, \\ \hat{\eta}_{t}+(\tilde{u} \hat{\eta})_{x}+\tilde{u}_{x}=0, & t>0, x \in \mathbb{R}, \\ \tilde{u}(0, x)=\tilde{u}_{0}(x) \quad \text { (odd), } & x \in \mathbb{R}, \\ \hat{\eta}(0, x)=\hat{\eta}_{0}(x) \quad \text { (even), } & x \in \mathbb{R} .\end{cases}
$$


Note that if the Green's function $p(x) \triangleq \frac{1}{2} e^{-|x|}, x \in \mathbb{R}$, then $\left(1-\partial_{x}^{2}\right)^{-1} f=p * f$ for all $f \in L^{2}(\mathbb{R})$. Using this identity, we can rewrite (3.4) as the quasilinear hyperbolic evolution equations

$$
\begin{cases}\tilde{u}_{t}+\tilde{u} \tilde{u}_{x}+\partial_{x} p *\left(\tilde{u}^{2}+\frac{1}{2} \tilde{u}_{x}^{2}+\frac{1}{2} \hat{\eta}^{2}+\hat{\eta}\right)=0, & t>0, x \in \mathbb{R}, \\ \hat{\eta}_{t}+(\tilde{u} \hat{\eta})_{x}+\tilde{u}_{x}=0, & t>0, x \in \mathbb{R}, \\ \tilde{u}(0, x)=\tilde{u}_{0}(x) \quad \text { (odd), } & x \in \mathbb{R}, \\ \hat{\eta}(0, x)=\hat{\eta}_{0}(x) \quad \text { (even), } & x \in \mathbb{R} .\end{cases}
$$

Applying the local well-posedness result for the Cauchy problem for $(2 \mathrm{CH})$ on the line obtained in [39], we conclude that there exists a maximal existence time $T=T\left(\tilde{u}_{0}, \hat{\eta}_{0}\right)>0$ such that the system (3.5) has a unique solution

$$
(\tilde{u}(t, x), \hat{\eta}(t, x)) \in E_{T}^{s, p}(\mathbb{R}) \times E_{T}^{s-1, p}(\mathbb{R}),
$$

and the solution depends continuously on the initial data, i.e., the mapping

$$
\left(\tilde{u}_{0}, \hat{\eta}_{0}\right) \mapsto(\tilde{u}, \hat{\eta}): V^{s, p}(\mathbb{R}) \times V^{s-1, p}(\mathbb{R}) \rightarrow E_{T}^{s, p}(\mathbb{R}) \times E_{T}^{s-1, p}(\mathbb{R})
$$

is continuous.

Moreover, the maximal $T$ is independent of $s$ in the following sense: if the solution $(\tilde{u}, \hat{\eta})$ to system $(3.5)$ belongs to $E_{T}^{s, 2}(\mathbb{R}) \times E_{T}^{s-1,2}(\mathbb{R})$ and if $\left(\tilde{u}_{0}, \hat{\eta}_{0}\right) \in$ $V^{s^{\prime}, 2}(\mathbb{R}) \times V^{s^{\prime}-1,2}(\mathbb{R})$ with $3 / 2<s \leq s^{\prime}<5 / 2$, then

$$
(\tilde{u}, \hat{\eta}) \in E_{T}^{s^{\prime}, 2}(\mathbb{R}) \times E_{T}^{s^{\prime}-1,2}(\mathbb{R})
$$

with the same $T$.

In addition, note that the system (3.4) is invariant under the transformation

$$
(\tilde{u}(t, x), \hat{\eta}(t, x)) \rightarrow(-\tilde{u}(t,-x), \hat{\eta}(t,-x)) .
$$

Since $\tilde{u}_{0}(x)$ is odd and $\hat{\eta}_{0}(x)$ is even, it follows that $\tilde{u}(t, x)$ is odd and $\hat{\eta}(t, x)$ is even for any $t \in[0, T)$. In particular, we have $\tilde{u}(t, 0) \equiv 0$ for all $t \in[0, T)$.

Set

$$
(u(t, x), \eta(t, x)) \triangleq(\tilde{u}(t, x), \hat{\eta}(t, x)), \quad \text { restricted to }(t, x) \in[0, T) \times \mathbb{R}_{+} .
$$

Hence,

$$
(u(t, x), \eta(t, x)) \in E_{T}^{s, p}\left(\mathbb{R}_{+}\right) \times E_{T}^{s-1, p}\left(\mathbb{R}_{+}\right)
$$

is a solution to (3.1).

On the other hand, if $(v(t, x), \gamma(t, x))$ is also a solution to (3.1) with the same initial data $\left(u_{0}(x), \eta_{0}(x)\right)$, set

$$
\tilde{v}(t, x)= \begin{cases}v(t, x), & \text { if } x \geq 0 \\ -v(t,-x), & \text { if } x<0\end{cases}
$$

and

$$
\hat{\gamma}(t, x)= \begin{cases}\gamma(t, x), & \text { if } x \geq 0 \\ \gamma(t,-x), & \text { if } x<0\end{cases}
$$


Then $(\tilde{v}(t, x), \hat{\gamma}(t, x))$ is also a solution to (3.5). By the uniqueness for the system (3.5), we conclude that

$$
(u(t, x), \eta(t, x))=(v(t, x), \gamma(t, x)) .
$$

It is obvious that the continuity of $\left(\tilde{u}_{0}, \hat{\eta}_{0}\right) \mapsto(\tilde{u}, \hat{\eta})$ implies that of $\left(u_{0}, \eta_{0}\right) \mapsto$ $(u, \eta)$ as well.

Remark 3.2. Assume that $\left(u_{0}, \eta_{0}\right) \in V^{s, p}\left(\mathbb{R}_{+}\right) \times V^{s-1, p}\left(\mathbb{R}_{+}\right)$with $1<p<\infty$ and $s \geq 2+1 / p$ satisfies the compatibility condition $u_{0}(0)=0$. If we fix some $r \in(\max (3 / 2,1+1 / p, 2-1 / p), 2+1 / p)$, then Theorem 3.1 implies that there exists a maximal $T=T\left(u_{0}, \eta_{0}\right)>0$ and a unique solution $(u(t, x), \eta(t, x)) \in$ $E_{T}^{r, p}\left(\mathbb{R}_{+}\right) \times E_{T}^{r-1, p}\left(\mathbb{R}_{+}\right)$to the system (3.1). However, one generally cannot deduce that $(u, \eta) \in E_{T}^{s, p}\left(\mathbb{R}_{+}\right) \times E_{T}^{s-1, p}\left(\mathbb{R}_{+}\right)$.

In order to study more regular solutions, we may consider the following initial boundary value problem for $k \in \mathbb{N}_{+}$:

$$
\begin{cases}u_{t}-u_{t x x}+3 u u_{x}=2 u_{x} u_{x x}+u u_{x x x}-\eta \eta_{x}-\eta_{x}, & t>0, x \in \mathbb{R}_{+}, \\ \eta_{t}+(u \eta)_{x}+u_{x}=0, & t>0, x \in \mathbb{R}_{+}, \\ u(0, x)=u_{0}(x), & x \in \mathbb{R}_{+}, \\ \eta(0, x)=\eta_{0}(x), & x \in \mathbb{R}_{+}, \\ u^{(2 k)}(t, 0)=u^{(2 k-2)}(t, 0)=\cdots=u(t, 0)=0, & t \geq 0, \\ \eta^{(2 k-1)}(t, 0)=\eta^{(2 k-3)}(t, 0)=\cdots=\eta^{\prime}(t, 0)=0, & t \geq 0 .\end{cases}
$$

We next present the following local well-posedness result.

Theorem 3.3. Let $k \in \mathbb{N}_{+}, 1<p<\infty$, and $2 k+1 / p<s<2 k+2+1 / p$. Assume that $\left(u_{0}, \eta_{0}\right) \in D_{k}^{s, p}\left(\mathbb{R}_{+}\right) \times R_{k}^{s-1, p}\left(\mathbb{R}_{+}\right)$. Then there exists a maximal $T=T\left(u_{0}, \eta_{0}\right)>0$ such that the system (3.8) has a unique solution $(u(t, x), \eta(t, x))$ in the space

$$
C\left([0, T) ; D_{k}^{s, p}\left(\mathbb{R}_{+}\right) \times R_{k}^{s-1, p}\left(\mathbb{R}_{+}\right)\right) \cap C^{1}\left([0, T) ; D_{k}^{s-1, p}\left(\mathbb{R}_{+}\right) \times R_{k}^{s-2, p}\left(\mathbb{R}_{+}\right)\right) .
$$

Moreover, the solution depends continuously on the initial data, i.e., the mapping $\left(u_{0}, \eta_{0}\right) \mapsto(u, \eta): D_{k}^{s, p}\left(\mathbb{R}_{+}\right) \times R_{k}^{s-1, p}\left(\mathbb{R}_{+}\right) \longrightarrow$

$$
C\left([0, T) ; D_{k}^{s, p}\left(\mathbb{R}_{+}\right) \times R_{k}^{s-1, p}\left(\mathbb{R}_{+}\right)\right) \cap C^{1}\left([0, T) ; D_{k}^{s-1, p}\left(\mathbb{R}_{+}\right) \times R_{k}^{s-2, p}\left(\mathbb{R}_{+}\right)\right)
$$

is continuous. Furthermore, the maximal $T$ is independent of $s$ in the following sense: if the solution $(u, \eta)$ to (3.8) belongs to

$$
C\left([0, T) ; D_{k}^{s, 2}\left(\mathbb{R}_{+}\right) \times R_{k}^{s-1,2}\left(\mathbb{R}_{+}\right)\right) \cap C^{1}\left([0, T) ; D_{k}^{s-1,2}\left(\mathbb{R}_{+}\right) \times R_{k}^{s-2,2}\left(\mathbb{R}_{+}\right)\right),
$$

and if $\left(u_{0}, \eta_{0}\right) \in D_{k}^{s^{\prime}, 2}\left(\mathbb{R}_{+}\right) \times R_{k}^{s^{\prime}-1,2}\left(\mathbb{R}_{+}\right)$with $2 k+1 / 2<s \leq s^{\prime}<2 k+5 / 2$, then $(u, \eta)$ belongs to

$$
C\left([0, T) ; D_{k}^{s^{\prime}, 2}\left(\mathbb{R}_{+}\right) \times R_{k}^{s^{\prime}-1,2}\left(\mathbb{R}_{+}\right)\right) \cap C^{1}\left([0, T) ; D_{k}^{s^{\prime}-1,2}\left(\mathbb{R}_{+}\right) \times R_{k}^{s^{\prime}-2,2}\left(\mathbb{R}_{+}\right)\right)
$$

with the same $T$. 
Proof. Arguing as in Theorem 3.1, we first extend the initial data $u_{0}(x)$ and $\eta_{0}(x)$ defined on the half line into the odd and even functions on the line $\tilde{u}_{0}(x)$ and $\hat{\eta}_{0}(x)$, defined in (3.2) and (3.3), respectively.

Since $\left(u_{0}, \eta_{0}\right) \in D_{k}^{s, p}\left(\mathbb{R}_{+}\right) \times R_{k}^{s-1, p}\left(\mathbb{R}_{+}\right)$, it follows from Lemmas 2.13 and 2.16 that

$$
\left(\tilde{u}_{0}(x), \hat{\eta}_{0}(x)\right) \in V^{s, p}(\mathbb{R}) \times V^{s-1, p}(\mathbb{R}) .
$$

The conclusions follow now as in Theorem 3.1.

Remark 3.4. From Lemmas 2.13 and 2.16, and Theorems 3.1 and 3.3, we see that $s=2 k+1 / p$ with $k \in \mathbb{N}_{+}$are the critical points for the problem (3.8).

Now we are in a position to present a precise blow-up scenario for strong solutions to the system (3.1).

Theorem 3.5. Let $\left(u_{0}, \eta_{0}\right) \in\left(H^{s}\left(\mathbb{R}_{+}\right) \cap H_{0}^{1}\left(\mathbb{R}_{+}\right)\right) \times H^{s-1}\left(\mathbb{R}_{+}\right)$with $3 / 2<s<5 / 2$, and let $(u(t, x), \eta(t, x))$ be the corresponding unique solution to (3.1). Then the solution $(u, \eta)$ blows up in finite time $T<+\infty$ if and only if

$$
\liminf _{t \uparrow T}\left\{\inf _{x \in \mathbb{R}_{+}} u_{x}(t, x)\right\}=-\infty .
$$

Proof. As before, we first extend the initial data $u_{0}(x)$ and $\eta_{0}(x)$ defined on the half line into the odd and even functions on the line $\tilde{u}_{0}(x)$ and $\hat{\eta}_{0}(x)$, defined in (3.2) and (3.3), respectively. By Theorem 3.1, we obtain that (3.5) has a unique strong solution $(\tilde{u}(t, x), \hat{\eta}(t, x))$ with the initial data $\left(\tilde{u}_{0}(x), \hat{\eta}_{0}(x)\right)$. Moreover, $(u(t, x), \eta(t, x)) \triangleq(\tilde{u}(t, x), \hat{\eta}(t, x))$ restricted to $[0, T) \times \mathbb{R}_{+}$is the unique strong solution to $(3.1)$ with the initial data $\left(u_{0}(x), \eta_{0}(x)\right)$.

From [40], we know that the solution $(\tilde{u}(t, x), \hat{\eta}(t, x))$ to the system (3.5) blows up in finite time $T$ if and only if

$$
\liminf _{t \uparrow T}\left\{\inf _{x \in \mathbb{R}} \tilde{u}_{x}(t, x)\right\}=-\infty .
$$

Since $\tilde{u}(t, \cdot)$ is odd, it follows that $\tilde{u}_{x}(t, \cdot)$ is even. Thus, we have

$$
\liminf _{t \uparrow T}\left\{\inf _{x \in \mathbb{R}} \tilde{u}_{x}(t, x)\right\}=\liminf _{t \uparrow T}\left\{\inf _{x \in \mathbb{R}_{+}} u_{x}(t, x)\right\},
$$

which implies the desired result.

Next, we state two blow-up results and one global existence result for (3.1).

Theorem 3.6. Let $\left(u_{0}, \eta_{0}\right) \in\left(H^{s}\left(\mathbb{R}_{+}\right) \cap H_{0}^{1}\left(\mathbb{R}_{+}\right)\right) \times H^{s-1}\left(\mathbb{R}_{+}\right)$with $3 / 2<s<5 / 2$. Assume that the initial data $\left(u_{0}(x), \eta_{0}(x)\right)$ satisfies

$$
u_{0}^{\prime}\left(x_{0}\right)<-\left(\left\|u_{0}\right\|_{H^{1}\left(\mathbb{R}_{+}\right)}^{2}+\left\|\eta_{0}\right\|_{L^{2}\left(\mathbb{R}_{+}\right)}^{2}\right)^{1 / 2} \quad \text { and } \quad \eta_{0}\left(x_{0}\right)=-1,
$$

with the point $x_{0} \in \mathbb{R}_{+}$defined by $u_{0}^{\prime}\left(x_{0}\right) \triangleq \inf _{x \in \mathbb{R}_{+}} u_{0}^{\prime}(x)$. Then the corresponding solution $(u, \eta)$ to $(3.1)$ blows up in finite time $T_{0}$, with

$$
0<T_{0} \leq-\frac{2}{(1-\delta) u_{0}^{\prime}\left(x_{0}\right)},
$$

where $\delta \in(0,1)$ is such that $-\sqrt{\delta} u_{0}^{\prime}\left(x_{0}\right)=\left(\left\|u_{0}\right\|_{H^{1}\left(\mathbb{R}_{+}\right)}^{2}+\left\|\eta_{0}\right\|_{L^{2}\left(\mathbb{R}_{+}\right)}^{2}\right)^{1 / 2}$. 
Proof. As mentioned earlier, let $\tilde{u}_{0}(x)$ and $\hat{\eta}_{0}(x)$ be defined as in (3.2) and (3.3). Since $\tilde{u}_{0}(x)$ is odd, $\tilde{u}_{0}^{\prime}(x)$ is even. By the assumption of the theorem and the fact

$$
\left\|\tilde{u}_{0}\right\|_{H^{1}(\mathbb{R})}^{2}+\left\|\hat{\eta}_{0}\right\|_{L^{2}(\mathbb{R})}^{2}=2\left(\left\|u_{0}\right\|_{H^{1}\left(\mathbb{R}_{+}\right)}^{2}+\left\|\eta_{0}\right\|_{L^{2}\left(\mathbb{R}_{+}\right)}^{2}\right),
$$

we can find some $x_{1} \in \mathbb{R}$ defined by

$$
\tilde{u}_{0}^{\prime}\left(x_{1}\right) \triangleq \inf _{x \in \mathbb{R}} \tilde{u}_{0}^{\prime}(x)=\inf _{x \in \mathbb{R}_{+}} u_{0}^{\prime}(x)=u_{0}^{\prime}\left(x_{0}\right),
$$

which satisfies

$$
\tilde{u}_{0}^{\prime}\left(x_{1}\right)<-\frac{1}{\sqrt{2}}\left(\left\|\tilde{u}_{0}\right\|_{H^{1}(\mathbb{R})}^{2}+\left\|\hat{\eta}_{0}\right\|_{L^{2}(\mathbb{R})}^{2}\right)^{1 / 2} \quad \text { and } \quad \hat{\eta}_{0}\left(x_{1}\right)=-1 .
$$

From [39], we get that the solution $(\tilde{u}, \hat{\eta})$ to $(3.5)$ with initial data $\left(\tilde{u}_{0}(x), \hat{\eta}_{0}(x)\right)$ blows up in finite time $T_{0}$ with

$$
0<T_{0} \leq-\frac{2}{(1-\delta) \tilde{u}_{0}^{\prime}\left(x_{1}\right)}
$$

where $\delta \in(0,1)$ is such that

$$
-\sqrt{\delta} \tilde{u}_{0}^{\prime}\left(x_{1}\right)=\frac{1}{\sqrt{2}}\left(\left\|\tilde{u}_{0}\right\|_{H^{1}(\mathbb{R})}^{2}+\left\|\hat{\eta}_{0}\right\|_{L^{2}(\mathbb{R})}^{2}\right)^{1 / 2} .
$$

This implies the desired result.

Theorem 3.7. Let $\left(u_{0}, \eta_{0}\right) \in\left(H^{s}\left(\mathbb{R}_{+}\right) \cap H_{0}^{1}\left(\mathbb{R}_{+}\right)\right) \times H^{s-1}\left(\mathbb{R}_{+}\right)$, with $3 / 2<s<5 / 2$. Assume that the initial data $\left(u_{0}, \eta_{0}\right)$ satisfies $u_{0}^{\prime}(0)<0$ and $\eta_{0}(0)=-1$. Then the corresponding solution to the system (3.1) blows up in finite time.

Proof. Let $\tilde{u}_{0}(x)$ and $\hat{\eta}_{0}(x)$ be defined as in (3.2) and (3.3). Then the assumption of the theorem ensures

$$
\tilde{u}_{0}^{\prime}(0)<0 \quad \text { and } \quad \hat{\eta}_{0}(0)=-1
$$

From [39], we know that the corresponding solution $(\tilde{u}, \hat{\eta})$ to $(3.5)$ blows up in finite time.

Since $\tilde{u}(t, \cdot)$ is odd, in view of Theorem 3.5 , it follows that the solution $(u, \eta)$ to $(3.1)$ with the initial data $\left(u_{0}(x), \eta_{0}(x)\right)$ blows up in finite time.

Theorem 3.8. Let $\left(u_{0}, \eta_{0}\right) \in\left(H^{s}\left(\mathbb{R}_{+}\right) \cap H_{0}^{1}\left(\mathbb{R}_{+}\right)\right) \times H^{s-1}\left(\mathbb{R}_{+}\right)$, with $3 / 2<s<5 / 2$, and let $T>0$ be the maximal existence time of the solution $(u, \eta)$ to system (3.1) with initial data $\left(u_{0}, \eta_{0}\right)$. If

$$
\inf _{x \in \mathbb{R}_{+}}\left(\eta_{0}(x)+1\right)>0
$$

then $T=\infty$, i.e., the solution $(u, \eta)$ exists globally in time. 
Proof. Let $\tilde{u}_{0}(x)$ and $\hat{\eta}_{0}(x)$ be defined as in (3.2) and (3.3). By the assumption of the theorem, since $\hat{\eta}_{0}(x)$ is even, we infer that

$$
\inf _{x \in \mathbb{R}}\left(\hat{\eta}_{0}(x)+1\right)>0 .
$$

From [40], we conclude that $T=\infty$.

Remark 3.9. Let $k \in \mathbb{N}_{+}$and $2 k+1 / 2<s<2 k+5 / 2$. Assume that $\left(u_{0}, \eta_{0}\right) \in$ $D_{k}^{s, 2}\left(\mathbb{R}_{+}\right) \times R_{k}^{s-1,2}\left(\mathbb{R}_{+}\right)$. Then Theorems $3.5-3.8$ also hold true for the corresponding solution $(u, \eta)$ to the system $(3.8)$.

We conclude this section with the existence of global weak solutions to (3.1).

Definition 3.10. Let $\left(u_{0}, \eta_{0}\right) \in H_{0}^{1}\left(\mathbb{R}_{+}\right) \times L^{2}\left(\mathbb{R}_{+}\right)$. If

$$
(u, \eta) \in L^{\infty}\left((0, \infty) ; H_{0}^{1}\left(\mathbb{R}_{+}\right) \times L^{2}\left(\mathbb{R}_{+}\right)\right)
$$

solves $(3.1)$ and $(u(t, \cdot), \eta(t, \cdot))$ converges to $\left(u_{0}(\cdot), \eta_{0}(\cdot)\right)$, in the sense of distributions, as $t$ tends to $0^{+}$, and moreover

$$
\|(u, \eta)\|_{L^{\infty}\left((0, \infty) ; H_{0}^{1}\left(\mathbb{R}_{+}\right) \times L^{2}\left(\mathbb{R}_{+}\right)\right)} \leq\left\|\left(u_{0}, \eta_{0}\right)\right\|_{H_{0}^{1}\left(\mathbb{R}_{+}\right) \times L^{2}\left(\mathbb{R}_{+}\right)},
$$

then $(u, \eta)$ is called a global admissible weak solution of (3.1).

Theorem 3.11. Let $\left(u_{0}, \eta_{0}\right) \in\left(H_{0}^{1}\left(\mathbb{R}_{+}\right) \cap W^{1, \infty}\left(\mathbb{R}_{+}\right)\right) \times\left(L^{2}\left(\mathbb{R}_{+}\right) \cap L^{\infty}\left(\mathbb{R}_{+}\right)\right)$. If there exists some $\alpha>-1$ such that $\eta_{0}(x) \geq \alpha$ for all $x \in \mathbb{R}_{+}$, then (3.1) has a global admissible weak solution

$$
(u, \eta) \in C\left(\mathbb{R}_{+} ; H_{0}^{1}\left(\mathbb{R}_{+}\right) \times L^{2}\left(\mathbb{R}_{+}\right)\right) \cap L^{\infty}\left(\mathbb{R}_{+} ; H_{0}^{1}\left(\mathbb{R}_{+}\right) \times L^{2}\left(\mathbb{R}_{+}\right)\right) .
$$

Moreover, $E(t) \triangleq\|(u(t, \cdot), \eta(t, \cdot))\|_{H^{1}\left(\mathbb{R}_{+}\right) \times L^{2}\left(\mathbb{R}_{+}\right)}^{2}$ is a conservation law. Furthermore,

$$
(u, \eta) \in L_{\text {loc }}^{\infty}\left(\mathbb{R}_{+} ; W^{1, \infty}\left(\mathbb{R}_{+}\right) \times L^{\infty}\left(\mathbb{R}_{+}\right)\right) .
$$

Proof. Let $\tilde{u}_{0}(x)$ and $\hat{\eta}_{0}(x)$ be defined as in (3.2) and (3.3). By the assumption of the theorem, in view of Lemmas 2.5 and 2.8, and Remarks 2.6 and 2.9, we obtain

$$
\left(\tilde{u}_{0}(x), \hat{\eta}_{0}(x)\right) \in\left(H^{1}(\mathbb{R}) \cap W^{1, \infty}(\mathbb{R})\right) \times\left(L^{2}(\mathbb{R}) \cap L^{\infty}(\mathbb{R})\right)
$$

and

$$
\hat{\eta}_{0}(x) \geq \alpha, \quad \forall x \in \mathbb{R} .
$$

From [37], we get that (3.5) has a global admissible weak solution

$$
(\tilde{u}, \hat{\eta}) \in C\left(\mathbb{R}_{+} ; H^{1}(\mathbb{R}) \times L^{2}(\mathbb{R})\right) \cap L^{\infty}\left(\mathbb{R}_{+} ; H^{1}(\mathbb{R}) \times L^{2}(\mathbb{R})\right) .
$$

Moreover, $E^{\star}(t) \triangleq\|(\tilde{u}(t, \cdot), \hat{\eta}(t, \cdot))\|_{H^{1}(\mathbb{R}) \times L^{2}(\mathbb{R})}^{2}$ is a conservation law. Furthermore,

$$
(\tilde{u}, \hat{\eta}) \in L_{\text {loc }}^{\infty}\left(\mathbb{R}_{+} ; W^{1, \infty}(\mathbb{R}) \times L^{\infty}(\mathbb{R})\right) .
$$

Set

$$
(u(t, x), \eta(t, x)) \triangleq(\tilde{u}(t, x), \hat{\eta}(t, x)), \quad \text { restricted to }(t, x) \in \mathbb{R}_{+} \times \mathbb{R}_{+} .
$$

Thus, $(u, \eta)$ is a global admissible weak solution to (3.1), which together with the fact $E^{\star}(t)=2 E(t)$ yield the desired result. 


\section{IBVP for the modified two-component Camassa-Holm shal- low water system}

In this section, we use the same method as in Sections 2 and 3 to deal with initial boundary value problems for the modified two-component Camassa-Holm shallow water system on the half line $(0, \infty)$.

Consider the initial boundary value problem for $(\mathrm{M} 2 \mathrm{CH})$ on the half line

$$
\begin{cases}m_{t}+u m_{x}+2 u_{x} m+\rho \gamma_{x}=0, & t>0, x \in \mathbb{R}_{+}, \\ \rho_{t}+(u \rho)_{x}=0, & t>0, x \in \mathbb{R}_{+}, \\ u(0, x)=u_{0}(x), & x \in \mathbb{R}_{+}, \\ \gamma(0, x)=\gamma_{0}(x), & x \in \mathbb{R}_{+}, \\ u(t, 0)=0, & t \geq 0, \\ \gamma(t, 0)=0, & t \geq 0,\end{cases}
$$

with $m=u-u_{x x}, \rho=\gamma-\gamma_{x x}$.

We first present the following local well-posedness result for (4.1).

Theorem 4.1. Assume that $\left(u_{0}, \gamma_{0}\right) \in H^{s}\left(\mathbb{R}_{+}\right) \times H^{s}\left(\mathbb{R}_{+}\right)$with $3 / 2<s<5 / 2$ satisfies the compatibility condition $u_{0}(0)=\gamma_{0}(0)=0$. Then there exists a maximal $T=T\left(u_{0}, \gamma_{0}\right)>0$ such that (4.1) has a unique solution $(u(t, x), \gamma(t, x)) \in$ $E_{T}^{s, 2}\left(\mathbb{R}_{+}\right) \times E_{T}^{s, 2}\left(\mathbb{R}_{+}\right)$.

Moreover, the solution depends continuously on the initial data, i.e., the mapping

$$
\left(u_{0}, \gamma_{0}\right) \mapsto(u, \gamma): H^{s}\left(\mathbb{R}_{+}\right) \times H^{s}\left(\mathbb{R}_{+}\right) \rightarrow E_{T}^{s, 2}\left(\mathbb{R}_{+}\right) \times E_{T}^{s, 2}\left(\mathbb{R}_{+}\right)
$$

is continuous. Furthermore, the maximal $T$ is independent of $s$ in the following sense: if $(u, \gamma) \in E_{T}^{s, 2}\left(\mathbb{R}_{+}\right) \times E_{T}^{s, 2}\left(\mathbb{R}_{+}\right)$is the solution to $(4.1)$, and if $\left(u_{0}, \gamma_{0}\right) \in$ $H^{s^{\prime}}\left(\mathbb{R}_{+}\right) \times H^{s^{\prime}}\left(\mathbb{R}_{+}\right)$with $3 / 2<s \leq s^{\prime}<5 / 2$ satisfies $u_{0}(0)=\gamma_{0}(0)=0$, then $(u, \gamma) \in E_{T}^{s^{\prime}, 2}\left(\mathbb{R}_{+}\right) \times E_{T}^{s^{\prime}, 2}\left(\mathbb{R}_{+}\right)$with the same $T$.

Proof. As before, we first convert (4.1) into the Cauchy problem for (M2CH) on the line. For this, we extend the initial data $\left(u_{0}(x), \gamma_{0}(x)\right)$ defined on the half line into odd functions defined on the line. That is (3.2) and

$$
\tilde{\gamma}_{0}(x) \triangleq \begin{cases}\gamma_{0}(x), & \text { if } x \geq 0 \\ -\gamma_{0}(-x), & \text { if } x<0\end{cases}
$$

By the assumption of the theorem and Lemma 2.11, one obtains

$$
\left(\tilde{u}_{0}(x), \tilde{\gamma}_{0}(x)\right) \in H^{s}(\mathbb{R}) \times H^{s}(\mathbb{R})
$$

with $3 / 2<s<5 / 2$.

Similar to the proof of Theorem 3.1, by using the Green's function, we can convert (4.1) into the following system on the whole line:

$$
\begin{cases}\tilde{u}_{t}+\tilde{u} \tilde{u}_{x}+\partial_{x} p *\left(\tilde{u}^{2}+\frac{1}{2} \tilde{u}_{x}^{2}+\frac{1}{2} \tilde{\gamma}^{2}-\frac{1}{2} \tilde{\gamma}_{x}^{2}\right)=0, & t>0, x \in \mathbb{R}, \\ \tilde{\gamma}_{t}+\tilde{u} \tilde{\gamma}_{x}+p *\left(\left(\tilde{u}_{x} \tilde{\gamma}_{x}\right)_{x}+\tilde{u}_{x} \tilde{\gamma}\right)=0, & t>0, x \in \mathbb{R}, \\ \tilde{u}(0, x)=\tilde{u}_{0}(x) \quad \text { (odd), } & x \in \mathbb{R}, \\ \tilde{\gamma}(0, x)=\tilde{\gamma}_{0}(x) \quad \text { (odd), } & x \in \mathbb{R} .\end{cases}
$$


Applying the local well-posedness result for the Cauchy problem for $(\mathrm{M} 2 \mathrm{CH})$ on the line obtained in [36], we conclude that there exists a maximal existence time $T=T\left(\tilde{u}_{0}, \tilde{\gamma}_{0}\right)>0$ such that the system (4.3) has a unique solution

$$
(\tilde{u}(t, x), \tilde{\gamma}(t, x)) \in E_{T}^{s, 2}(\mathbb{R}) \times E_{T}^{s-1,2}(\mathbb{R}),
$$

and the solution depends continuously on the initial data, i.e., the mapping

$$
\left(\tilde{u}_{0}, \tilde{\gamma}_{0}\right) \mapsto(\tilde{u}, \tilde{\gamma}): H^{s}(\mathbb{R}) \times H^{s}(\mathbb{R}) \rightarrow E_{T}^{s, 2}(\mathbb{R}) \times E_{T}^{s-1,2}(\mathbb{R})
$$

is continuous. Moreover, the maximal $T$ is independent of $s$ in the following sense: if the solution $(\tilde{u}, \tilde{\gamma})$ to system $(4.3)$ belongs to $E_{T}^{s, 2}(\mathbb{R}) \times E_{T}^{s-1,2}(\mathbb{R})$, and if $\left(\tilde{u}_{0}, \tilde{\gamma}_{0}\right) \in H^{s^{\prime}}(\mathbb{R}) \times H^{s^{\prime}}(\mathbb{R})$ with $3 / 2<s \leq s^{\prime}<5 / 2$, then

$$
(\tilde{u}, \tilde{\gamma}) \in E_{T}^{s^{\prime}, 2}(\mathbb{R}) \times E_{T}^{s^{\prime}-1,2}(\mathbb{R})
$$

with the same $T$. In addition, note that (4.3) is invariant under the transformation

$$
(\tilde{u}(t, x), \tilde{\gamma}(t, x)) \rightarrow(-\tilde{u}(t,-x),-\tilde{\gamma}(t,-x)) .
$$

Since $\tilde{u}_{0}(x)$ and $\tilde{\gamma}_{0}(x)$ are odd, it follows that $\tilde{u}(t, x)$ and $\tilde{\gamma}(t, x)$ are odd for any $t \in[0, T)$. In particular, we have $\tilde{u}(t, 0)=\tilde{\gamma}(t, 0) \equiv 0$ for all $t \in[0, T)$.

Set

$$
(u(t, x), \gamma(t, x)) \triangleq(\tilde{u}(t, x), \tilde{\gamma}(t, x)), \quad \text { restricted to }(t, x) \in[0, T) \times \mathbb{R}_{+} .
$$

Hence,

$$
(u(t, x), \gamma(t, x)) \in E_{T}^{s, 2}\left(\mathbb{R}_{+}\right) \times E_{T}^{s-1,2}\left(\mathbb{R}_{+}\right)
$$

is a solution to (4.1).

On the other hand, if $(v(t, x), \rho(t, x))$ is also a solution to (4.1) with the same initial data $\left(u_{0}(x), \gamma_{0}(x)\right)$, set

$$
\tilde{\rho}(t, x)= \begin{cases}\rho(t, x), & \text { if } x \geq 0, \\ -\rho(t,-x), & \text { if } x<0, .\end{cases}
$$

This together with 3.6 yield $(\tilde{v}(t, x), \tilde{\rho}(t, x))$ is also a solution of (4.3). By the uniqueness for the system (4.3), we conclude that

$$
(u(t, x), \gamma(t, x))=(v(t, x), \rho(t, x)) .
$$

It is obvious that the continuity of $\left(\tilde{u}_{0}, \tilde{\gamma}_{0}\right) \mapsto(\tilde{u}, \tilde{\gamma})$ implies that of $\left(u_{0}, \gamma_{0}\right) \mapsto$ $(u, \gamma)$ as well.

In order to obtain higher regularity of of the solutions, we consider the following initial boundary value problem:

$$
\begin{cases}m_{t}+u m_{x}+2 u_{x} m+\rho \gamma_{x}=0, & t>0, x \in \mathbb{R}_{+}, \\ \rho_{t}+(u \rho)_{x}=0, & t>0, x \in \mathbb{R}_{+}, \\ u(0, x)=u_{0}(x), & x \in \mathbb{R}_{+}, \\ \gamma(0, x)=\gamma_{0}(x), & x \in \mathbb{R}_{+}, \\ u^{(2 k)}(t, 0)=u^{(2 k-2)}(t, 0)=\cdots=u(t, 0)=0, & t \geq 0, \\ \gamma^{(2 k)}(t, 0)=\gamma^{(2 k-2)}(t, 0)=\cdots=\gamma(t, 0)=0, & t \geq 0,\end{cases}
$$

with $m=u-u_{x x}, \rho=\gamma-\gamma_{x x}$. 
Similar to Theorem 3.3, we have the following result:

Theorem 4.2. Let $k \in \mathbb{N}_{+}$and $2 k+1 / 2<s<2 k+5 / 2$. Assume that $\left(u_{0}, \gamma_{0}\right) \in$ $D_{k}^{s, 2}\left(\mathbb{R}_{+}\right) \times D_{k}^{s, 2}\left(\mathbb{R}_{+}\right)$. Then there exists a maximal $T=T\left(u_{0}, \gamma_{0}\right)>0$ such that (4.4) has a unique solution $(u(t, x), \gamma(t, x))$ in the space

$$
C\left([0, T) ; D_{k}^{s, 2}\left(\mathbb{R}_{+}\right) \times D_{k}^{s, 2}\left(\mathbb{R}_{+}\right)\right) \cap C^{1}\left([0, T) ; D_{k}^{s-1,2}\left(\mathbb{R}_{+}\right) \times D_{k}^{s-1,2}\left(\mathbb{R}_{+}\right)\right) .
$$

Moreover, the solution depends continuously on the initial data, i.e., the mapping $\left(u_{0}, \gamma_{0}\right) \mapsto(u, \gamma): D_{k}^{s, 2}\left(\mathbb{R}_{+}\right) \times D_{k}^{s, 2}\left(\mathbb{R}_{+}\right) \longrightarrow$

$$
C\left([0, T) ; D_{k}^{s, 2}\left(\mathbb{R}_{+}\right) \times D_{k}^{s, 2}\left(\mathbb{R}_{+}\right)\right) \cap C^{1}\left([0, T) ; D_{k}^{s-1,2}\left(\mathbb{R}_{+}\right) \times D_{k}^{s-1,2}\left(\mathbb{R}_{+}\right)\right) .
$$

is continuous. Furthermore, the maximal $T$ is independent of $s$ in the following sense: if the solution $(u, \gamma)$ to (4.4) belongs to

$$
C\left([0, T) ; D_{k}^{s, 2}\left(\mathbb{R}_{+}\right) \times D_{k}^{s, 2}\left(\mathbb{R}_{+}\right)\right) \cap C^{1}\left([0, T) ; D_{k}^{s-1,2}\left(\mathbb{R}_{+}\right) \times D_{k}^{s-1,2}\left(\mathbb{R}_{+}\right)\right),
$$

and if $\left(u_{0}, \gamma_{0}\right) \in D_{k}^{s^{\prime}, 2}\left(\mathbb{R}_{+}\right) \times D_{k}^{s^{\prime}, 2}\left(\mathbb{R}_{+}\right)$with $2 k+1 / 2<s \leq s^{\prime}<2 k+5 / 2$, then $(u, \gamma)$ belongs to

$$
C\left([0, T) ; D_{k}^{s^{\prime}, 2}\left(\mathbb{R}_{+}\right) \times D_{k}^{s^{\prime}, 2}\left(\mathbb{R}_{+}\right)\right) \cap C^{1}\left([0, T) ; D_{k}^{s^{\prime}-1,2}\left(\mathbb{R}_{+}\right) \times D_{k}^{s^{\prime}-1,2}\left(\mathbb{R}_{+}\right)\right)
$$

with the same $T$.

Remark 4.3. From Lemma 2.13 and Theorems 4.1 and 4.2 , we see that the critical points for the problem (4.4) are $s=2 k+1 / 2$, with $k \in \mathbb{N}_{+}$.

Now we are in a position to present a precise blow-up scenario for strong solutions to (4.1).

Theorem 4.4. Let $\left(u_{0}, \gamma_{0}\right) \in\left(H^{s}\left(\mathbb{R}_{+}\right) \cap H_{0}^{1}\left(\mathbb{R}_{+}\right)\right) \times\left(H^{s}\left(\mathbb{R}_{+}\right) \cap H_{0}^{1}\left(\mathbb{R}_{+}\right)\right)$, with $3 / 2<s<5 / 2$, and denote by $T$ the maximal existence time of the solution $(u(t, x), \gamma(t, x))$ of (4.1) with initial data $\left(u_{0}, \gamma_{0}\right)$. Then $T$ is finite if and only if

$$
\liminf _{t \uparrow T}\left\{\inf _{x \in \mathbb{R}_{+}} u_{x}(t, x)\right\}=-\infty .
$$

Proof. As before, we first extend the initial data $\left(u_{0}(x), \gamma_{0}(x)\right)$ defined on the half line into the odd functions on the line $\left(\tilde{u}_{0}(x), \tilde{\gamma}_{0}(x)\right)$ defined in (3.2) and (4.2), respectively. By Theorem 4.1, we obtain that (4.3) has a unique strong solution $(\tilde{u}(t, x), \tilde{\gamma}(t, x))$ with the initial data $\left(\tilde{u}_{0}(x), \tilde{\gamma}_{0}(x)\right)$. Moreover, $(u(t, x), \gamma(t, x)) \triangleq$ $(\tilde{u}(t, x), \tilde{\gamma}(t, x))$ restricted to $[0, T) \times \mathbb{R}_{+}$is the unique strong solution to the system (4.1) with the initial data $\left(u_{0}(x), \gamma_{0}(x)\right)$.

From [36], we know that the solution $(\tilde{u}(t, x), \tilde{\gamma}(t, x))$ to (4.3) blows up in finite time $T<+\infty$ if and only if

$$
\liminf _{t \uparrow T}\left\{\inf _{x \in \mathbb{R}} \tilde{u}_{x}(t, x)\right\}=-\infty .
$$

Since $\tilde{u}(t, \cdot)$ is odd, it follows that (3.9) is true, which implies the desired result. 
Next, as we did in Section 3, by using blow-up results for (1.2) established previously in [36], one can easily obtain the following blow-up theorem.

Theorem 4.5. Let $\left(u_{0}, \gamma_{0}\right) \in\left(H^{s}\left(\mathbb{R}_{+}\right) \cap H_{0}^{1}\left(\mathbb{R}_{+}\right)\right) \times\left(H^{s}\left(\mathbb{R}_{+}\right) \cap H_{0}^{1}\left(\mathbb{R}_{+}\right)\right)$, with $3 / 2<s<5 / 2$, and denote by $T$ the maximal existence time of the solution $(u(t, x), \gamma(t, x))$ to (4.1) with the initial data $\left(u_{0}, \gamma_{0}\right)$. If there exists some $x_{0} \in \mathbb{R}_{+}$ such that

or if

$$
u_{0}^{\prime}\left(x_{0}\right)<\sqrt{2}\left(\left\|u_{0}\right\|_{H^{1}\left(\mathbb{R}_{+}\right)}^{2}+\left\|\gamma_{0}\right\|_{H^{1}\left(\mathbb{R}_{+}\right)}^{2}\right)^{1 / 2},
$$

then $T$ is finite.

$$
u_{0}^{\prime}(0)<\left(\left\|u_{0}\right\|_{H^{1}\left(\mathbb{R}_{+}\right)}^{2}+\left\|\gamma_{0}\right\|_{H^{1}\left(\mathbb{R}_{+}\right)}^{2}\right)^{1 / 2}
$$

Remark 4.6. Let $k \in \mathbb{N}_{+}$and $2 k+1 / 2<s<2 k+5 / 2$. Assume that $\left(u_{0}, \gamma_{0}\right) \in$ $D_{k}^{s, 2}\left(\mathbb{R}_{+}\right) \times D_{k}^{s, 2}\left(\mathbb{R}_{+}\right)$. Then Theorems 4.4 and 4.5 also hold true for the corresponding solution $(u, \gamma)$ to $(4.4)$.

We conclude this section with the existence of global weak solutions to (4.1).

Definition 4.7. By a solution to $(4.1)$ on $\left[t_{1}, t_{2}\right]$ we mean a pair of Hölder continuous functions $(u(t, x), \gamma(t, x))$ defined on $\left[t_{1}, t_{2}\right] \times \mathbb{R}_{+}$with the following properties: at each fixed time $t$,

$$
(u(t, \cdot), \gamma(t, \cdot)) \in H_{0}^{1}\left(\mathbb{R}_{+}\right) \times\left(H_{0}^{1}\left(\mathbb{R}_{+}\right) \cap W^{1, \infty}\left(\mathbb{R}_{+}\right)\right) .
$$

Moreover, the maps $u(t, \cdot)$ and $\gamma(t, \cdot)$ are both Lipschitz continuous from $\left[t_{1}, t_{2}\right]$ to $L^{2}\left(\mathbb{R}_{+}\right)$, and satisfy $(4.1)$ in the $L^{2}\left(\mathbb{R}_{+}\right)$sense. Furthermore, if the energy

$$
E(t) \triangleq\|u(t, \cdot)\|_{H^{1}\left(\mathbb{R}_{+}\right)}^{2}+\|\gamma(t, \cdot)\|_{H^{1}\left(\mathbb{R}_{+}\right)}^{2}
$$

is a nonincreasing function of $t$, then $(u, \gamma)$ is called a dissipative solution to (4.1). If $E(t)$ is a conservation law, we call $(u, \gamma)$ a conservative solution to (4.1).

Theorem 4.8. Assume that $\left(u_{0}, \gamma_{0}\right) \in H_{0}^{1}\left(\mathbb{R}_{+}\right) \times\left(H_{0}^{1}\left(\mathbb{R}_{+}\right) \cap W^{1, \infty}\left(\mathbb{R}_{+}\right)\right)$. Then the system (4.1) has a solution, defined for any given time interval $[0, T]$, in the sense of Definition 4.7. That is, (4.1) has a global dissipative solution and a global conservative solution.

Proof. Let $\tilde{u}_{0}(x)$ and $\tilde{\gamma}_{0}(x)$ be defined as in (3.2) and (4.2). By the assumption of the theorem, in view of Lemma 2.5 and Remark 2.6, we obtain that

$$
\left(\tilde{u}_{0}(x), \tilde{\gamma}_{0}(x)\right) \in H^{1}(\mathbb{R}) \times\left(H^{1}(\mathbb{R}) \cap W^{1, \infty}(\mathbb{R})\right)
$$

From [56] and [57], we get that (4.3) has a global dissipative solution and a global conservative solution, defined on $\mathbb{R}_{+} \times \mathbb{R}$.

Set

$$
(u(t, x), \gamma(t, x)) \triangleq(\tilde{u}(t, x), \tilde{\gamma}(t, x)), \quad \text { restricted to }(t, x) \in \mathbb{R}_{+} \times \mathbb{R}_{+} .
$$

This together with the fact that

$$
\|\tilde{u}(t, \cdot)\|_{H^{1}(\mathbb{R})}^{2}+\|\tilde{\gamma}(t, \cdot)\|_{H^{1}(\mathbb{R})}^{2}=2\left(\|u(t, \cdot)\|_{H^{1}\left(\mathbb{R}_{+}\right)}^{2}+\|\gamma(t, \cdot)\|_{H^{1}\left(\mathbb{R}_{+}\right)}^{2}\right)
$$

yields the desired result. 


\section{IBVP for two-component Degasperis-Procesi system}

In this section, we use the method established in Sections 2-4 to deal with initial boundary value problems for the two-component Degasperis-Procesi system on the half line $(0, \infty)$. line:

Consider following the initial boundary value problems for (2DP) on the half

$$
\begin{cases}u_{t}-u_{t x x}+4 u u_{x}-3 u_{x} u_{x x}-u u_{x x x}+c \eta \eta_{x}=0, & t>0, x \in \mathbb{R}_{+}, \\ \eta_{t}+u \eta_{x}+2 u_{x} \eta=0, & t>0, x \in \mathbb{R}_{+}, \\ u(0, x)=u_{0}(x), & x \in \mathbb{R}_{+}, \\ \eta(0, x)=\eta_{0}(x), & x \in \mathbb{R}_{+}, \\ u(t, 0)=0, & t \geq 0 .\end{cases}
$$

We first present the following local well-posedness result for (5.1).

Theorem 5.1. Let $1<p<\infty$ and $\max (3 / 2,1+1 / p, 2-1 / p)<s<2+1 / p$. Assume that $\left(u_{0}, \eta_{0}\right) \in V^{s, p}\left(\mathbb{R}_{+}\right) \times V^{s-1, p}\left(\mathbb{R}_{+}\right)$satisfies the compatibility condition $u_{0}(0)=0$. Then there exists a maximal $T=T\left(u_{0}, \eta_{0}\right)>0$ such that (5.1) has a unique solution $(u(t, x), \eta(t, x)) \in E_{T}^{s, p}\left(\mathbb{R}_{+}\right) \times E_{T}^{s-1, p}\left(\mathbb{R}_{+}\right)$. Moreover, the solution depends continuously on the initial data, i.e., the mapping

$$
\left(u_{0}, \eta_{0}\right) \mapsto(u, \eta): V^{s, p}\left(\mathbb{R}_{+}\right) \times V^{s-1, p}\left(\mathbb{R}_{+}\right) \rightarrow E_{T}^{s, p}\left(\mathbb{R}_{+}\right) \times E_{T}^{s-1, p}\left(\mathbb{R}_{+}\right)
$$

is continuous. Furthermore, the maximal $T$ is independent of $s$ in the following sense: if $(u, \eta) \in E_{T}^{s, 2}\left(\mathbb{R}_{+}\right) \times E_{T}^{s-1,2}\left(\mathbb{R}_{+}\right)$is the solution to (5.1), and if $\left(u_{0}, \eta_{0}\right) \in$ $H^{s^{\prime}}\left(\mathbb{R}_{+}\right) \times H^{s^{\prime}-1}\left(\mathbb{R}_{+}\right)$with $3 / 2<s \leq s^{\prime}<5 / 2$ satisfies $u_{0}(0)=0$, then $(u, \eta) \in$ $E_{T}^{s^{\prime}, 2}\left(\mathbb{R}_{+}\right) \times E_{T}^{s^{\prime}-1,2}\left(\mathbb{R}_{+}\right)$with the same $T$.

Proof. As in the proof of Theorem 3.1, let $\tilde{u}_{0}(x)$ and $\hat{\eta}_{0}(x)$ be defined as in $(3.2)$ and (3.3). Then

$$
\left(\tilde{u}_{0}(x), \hat{\eta}_{0}(x)\right) \in V^{s, p}(\mathbb{R}) \times V^{s-1, p}(\mathbb{R})
$$

with $\max (3 / 2,1+1 / p, 2-1 / p)<s<2+1 / p$.

By using the Green's function, we can also convert (5.1) into the following system on the whole line:

$$
\begin{cases}\tilde{u}_{t}+\tilde{u} \tilde{u}_{x}+\partial_{x} p *\left(3 / 2 \tilde{u}^{2}+\frac{c}{2} \hat{\eta}^{2}\right)=0, & t>0, x \in \mathbb{R}, \\ \hat{\eta}_{t}+\tilde{u} \hat{\eta}_{x}+2 \tilde{u}_{x} \eta=0, & t>0, x \in \mathbb{R}, \\ \tilde{u}(0, x)=\tilde{u}_{0}(x) \quad \text { (odd), } & x \in \mathbb{R}, \\ \hat{\eta}(0, x)=\hat{\eta}_{0}(x) \quad \text { (even), } & x \in \mathbb{R} .\end{cases}
$$

Applying the local well-posedness result for the Cauchy problem for (2DP) on the line established previously in [65], and following the lines of the proof of Theorem 3.1, we can easily prove the theorem. 
In order to obtain solutions with higher regularity, let us consider the following initial boundary value problem:

$$
\begin{cases}u_{t}-u_{t x x}+4 u u_{x}-3 u_{x} u_{x x}-u u_{x x x}+c \eta \eta_{x}=0, & t>0, x \in \mathbb{R}_{+} \\ \eta_{t}+u \eta_{x}+2 u_{x} \eta=0, & t>0, x \in \mathbb{R}_{+}, \\ u(0, x)=u_{0}(x), & x \in \mathbb{R}_{+} \\ \eta(0, x)=\eta_{0}(x), & x \in \mathbb{R}_{+} \\ u^{(2 k)}(t, 0)=u^{(2 k-2)}(t, 0)=\cdots=u(t, 0)=0, & t \geq 0 \\ \eta^{(2 k-1)}(t, 0)=\eta^{(2 k-3)}(t, 0)=\cdots=\eta^{\prime}(t, 0)=0, & t \geq 0\end{cases}
$$

We have the following result, similar to Theorem 3.3:

Theorem 5.2. Let $k \in \mathbb{N}_{+}, 1<p<\infty$ and $2 k+1 / p<s<2 k+2+1 / p$. Assume that $\left(u_{0}, \eta_{0}\right) \in D_{k}^{s, p}\left(\mathbb{R}_{+}\right) \times R_{k}^{s-1, p}\left(\mathbb{R}_{+}\right)$. Then there exists a maximal $T=T\left(u_{0}, \eta_{0}\right)>0$ such that (5.3) has a unique solution $(u(t, x), \eta(t, x))$ in the space

$$
C\left([0, T) ; D_{k}^{s, p}\left(\mathbb{R}_{+}\right) \times R_{k}^{s-1, p}\left(\mathbb{R}_{+}\right)\right) \cap C^{1}\left([0, T) ; D_{k}^{s-1, p}\left(\mathbb{R}_{+}\right) \times R_{k}^{s-2, p}\left(\mathbb{R}_{+}\right)\right) .
$$

Moreover, the solution depends continuously on the initial data, i.e., the mapping $\left(u_{0}, \eta_{0}\right) \mapsto(u, \eta): D_{k}^{s, p}\left(\mathbb{R}_{+}\right) \times R_{k}^{s-1, p}\left(\mathbb{R}_{+}\right) \longrightarrow$

$$
C\left([0, T) ; D_{k}^{s, p}\left(\mathbb{R}_{+}\right) \times R_{k}^{s-1, p}\left(\mathbb{R}_{+}\right)\right) \cap C^{1}\left([0, T) ; D_{k}^{s-1, p}\left(\mathbb{R}_{+}\right) \times R_{k}^{s-2, p}\left(\mathbb{R}_{+}\right)\right)
$$

is continuous. Furthermore, the maximal $T$ is independent of $s$ in the following sense: if the solution $(u, \eta)$ to $(5.3)$ belongs to

$$
C\left([0, T) ; D_{k}^{s, 2}\left(\mathbb{R}_{+}\right) \times R_{k}^{s-1,2}\left(\mathbb{R}_{+}\right)\right) \cap C^{1}\left([0, T) ; D_{k}^{s-1,2}\left(\mathbb{R}_{+}\right) \times R_{k}^{s-2,2}\left(\mathbb{R}_{+}\right)\right),
$$

and if $\left(u_{0}, \eta_{0}\right) \in D_{k}^{s^{\prime}, 2}\left(\mathbb{R}_{+}\right) \times R_{k}^{s^{\prime}-1,2}\left(\mathbb{R}_{+}\right)$with $2 k+1 / 2<s \leq s^{\prime}<2 k+5 / 2$, then $(u, \eta)$ belongs to

$$
C\left([0, T) ; D_{k}^{s^{\prime}, 2}\left(\mathbb{R}_{+}\right) \times R_{k}^{s^{\prime}-1,2}\left(\mathbb{R}_{+}\right)\right) \cap C^{1}\left([0, T) ; D_{k}^{s^{\prime}-1,2}\left(\mathbb{R}_{+}\right) \times R_{k}^{s^{\prime}-2,2}\left(\mathbb{R}_{+}\right)\right)
$$

with the same $T$.

Remark 5.3. From Lemmas 2.13 and 2.16, and Theorems 5.1 and 5.2, we see that $s=2 k+1 / p$, with $k \in \mathbb{N}_{+}$, are the critical points for the problem (5.3).

Next, similar to Section 3 and by using the previous blow-up results of (1.3) in [65], one can easily obtain the following results about (5.1).

Theorem 5.4. Let $\left(u_{0}, \eta_{0}\right) \in\left(H^{s}\left(\mathbb{R}_{+}\right) \cap H_{0}^{1}\left(\mathbb{R}_{+}\right)\right) \times H^{s-1}\left(\mathbb{R}_{+}\right)$with $3 / 2<s<5 / 2$, and let $(u(t, x), \eta(t, x))$ be the corresponding unique solution of (5.1). Then the solution $(u, \eta)$ blows up in finite time $T<+\infty$ if and only if

$$
\liminf _{t \uparrow T}\left\{\inf _{x \in \mathbb{R}_{+}} u_{x}(t, x)\right\}=-\infty .
$$


Theorem 5.5. Let $\left(u_{0}, \eta_{0}\right) \in\left(H^{s}\left(\mathbb{R}_{+}\right) \cap H_{0}^{1}\left(\mathbb{R}_{+}\right)\right) \times H^{s-1}\left(\mathbb{R}_{+}\right)$, with $3 / 2<s<5 / 2$. Assume that $c \geq 0, u_{0}^{\prime}(0)<0$ and $\eta_{0}(0)=0$. Then the corresponding solution $(u, \eta)$ of $(5.1)$ blows up in finite time $T$, with $0<T \leq-1 / u_{0}^{\prime}(0)$.

Remark 5.6. Let $k \in \mathbb{N}_{+}$and $2 k+1 / 2<s<2 k+5 / 2$. Assume that $\left(u_{0}, \eta_{0}\right) \in$ $D_{k}^{s, 2}\left(\mathbb{R}_{+}\right) \times R_{k}^{s-1,2}\left(\mathbb{R}_{+}\right)$. Then Theorems 5.4 and 5.5 also hold true for the corresponding solution $(u, \eta)$ to $(5.3)$.

\section{References}

[1] Adams, R. A.: Sobolev spaces. Pure and Applied Mathematics 65, Academic Press, New York-London, 1975.

[2] Beals, R., Sattinger, D. and Szmigielski, J.: Acoustic scattering and the extended Korteweg-de Vries hierarchy. Adv. Math. 140 (1998), 190-206.

[3] Bressan, A. And Constantin, A.: Global dissipative solutions of the CamassaHolm equation. Anal. Appl. (Singap.) 5 (2007), 1-27.

[4] Bressan, A. and Constantin, A.: Global conservative solutions of the CamassaHolm equation. Arch. Ration. Mech. Anal. 183 (2007), 215-239.

[5] Camassa, R. And Holm, D.: An integrable shallow water equation with peaked solitons. Phys. Rev. Lett. 71 (1993), 1661-1664.

[6] Camassa, R., Holm, D. And Hyman, J.: A new integrable shallow water equation. Adv. in Appl. Mech. 31 (1994), 1-33.

[7] Chen, M., Liu, S.-Q. And Zhang, Y.: A two-component generalization of the Camassa-Holm equation and its solutions. Lett. Math. Phys. 75 (2006), 1-15.

[8] Coclite, G. M. And Karlsen, K. H.: On the well-posedness of the DegasperisProcesi equation. J. Funct. Anal. 233 (2006), 60-91.

[9] Constantin, A.: Existence of permanent and breaking waves for a shallow water equation: a geometric approach. Ann. Inst. Fourier (Grenoble) 50 (2000), 321-362.

[10] Constantin, A.: On the scattering problem for the Camassa-Holm equation. Proc. Roy. Soc. London Ser. A 457 (2001), 953-970.

[11] Constantin, A.: The trajectories of particles in Stokes waves. Invent. Math. 166 (2006), 523-535.

[12] Constantin, A. And Escher, J.: Global existence and blow-up for a shallow water equation. Ann. Scuola Norm. Sup. Pisa Cl. Sci. (4) 26 (1998), 303-328.

[13] Constantin, A. And Escher, J.: Well-posedness, global existence, and blowup phenomena for a periodic quasi-linear hyperbolic equation. Comm. Pure Appl. Math. 51 (1998), 475-504.

[14] Constantin, A. And Escher, J.: Wave breaking for nonlinear nonlocal shallow water equations. Acta Math. 181 (1998), 229-243.

[15] Constantin, A. And Escher, J.: Analyticity of periodic traveling free surface water waves with vorticity. Ann. of Math. (2) 173 (2011), 559-568.

[16] Constantin, A. And Ivanov, R.: On an integrable two-component Camassa-Holm shallow water system. Phys. Lett. A 372 (2008), 7129-7132.

[17] Constantin, A., Ivanov, R. And Lenells, J.: Inverse scattering transform for the Degasperis-Procesi equation. Nonlinearity 23 (2010), 2559-2575. 
[18] Constantin, A. And Kolev, B.: Geodesic flow on the diffeomorphism group of the circle. Comm. Math. Helv. 78 (2003), 787-804.

[19] Constantin, A. And Lannes, D.: The hydrodynamical relevance of the CamassaHolm and Degasperis-Procesi equations. Arch. Ration. Mech. Anal. 192 (2009), $165-186$.

[20] Constantin, A. And Molinet, L.: Global weak solutions for a shallow water equation. Comm. Math. Phys. 211 (2000), 45-61.

[21] Constantin, A. And Strauss, W. A.: Stability of peakons. Comm. Pure Appl. Math. 53 (2000), 603-610.

[22] DAI, H.-H.: Model equations for nonlinear dispersive waves in a compressible Mooney-Rivlin rod. Acta Mech. 127 (1998), 193-207.

[23] Danchin, R.: A note on well-posedness for Camassa-Holm equation. J. Differential Equations 192 (2003), 429-444.

[24] Degasperis, A., Holm, D. D. And Hone, A. N. W.: A new integrable equation with peakon solutions. Theoret. and Math. Phys. 133 (2002), 1463-1474.

[25] Degasperis, A. And Procesi, M.: Asymptotic integrability. In Symmetry and perturbation theory (Rome, 1998), 23-37. World Sci. Publishing, River Edge, NJ, 1999 .

[26] Dullin, H. R., Gottwald, G. A. And Holm, D. D.: An integrable shallow water equation with linear and nonlinear dispersion. Phys. Rev. Lett. 87 (2001), 4501-4504.

[27] Escher, J. And Kolev, B.: The Degasperis-Procesi equation as a non-metric Euler equation. Math. Z. 269 (2011), 1137-1153.

[28] Escher, J., Lechtenfeld, O. And Yin, Z.: Well-posedness and blow-up phenomena for the 2-component Camassa-Holm equation. Discrete Contin. Dyn. Syst. 19 (2007), 493-513.

[29] Escher, J., Liu, Y. ANd Yin, Z.: Global weak solutions and blow-up structure for the Degasperis-Procesi equation. J. Funct. Anal. 241 (2006), 457-485.

[30] Escher, J., Liu, Y. And Yin, Z.: Shock waves and blow-up phenomena for the periodic Degasperis-Procesi equation. Indiana Univ. Math. J. 56 (2007), 87-117.

[31] Escher, J. AND Yin, Z.: Initial boundary value problems for nonlinear dispersive wave equations. J. Funct. Anal. 256 (2009), 479-508.

[32] FAlqui, G.: On a Camassa-Holm type equation with two dependent variables. J. Phys. A 39 (2006), 327-342.

[33] Fokas, A. And Fuchssteiner, B.: Symplectic structures, their Bäcklund transformation and hereditary symmetries. Phys. D 4 (1981), 47-66.

[34] Gilbarg, D. and Trudinger, N. S.: Elliptic partial differential equations of second order. Grundlehren der Mathematischen Wissenschaften 224, Springer-Verlag, Berlin-New York, 1977.

[35] Guan, C. And Yin, Z.: Global existence and blow-up phenomena for an integrable two-component Camassa-Holm shallow water system. J. Differential Equations 248 (2010), 2003-2014.

[36] Guan, C., Karlsen, K. H. And Yin, Z.: Well-posedness and blow-up phenomena for a modified two-component Camassa-Holm equation. In Nonlinear partial differential equations and hyperbolic wave phenomena., 199-220. Contemp. Math. 526, Amer. Math. Soc., Providence, RI, 2010. 
[37] Guan, C. And Yin, Z.: Global weak solutions for a two-component Camassa-Holm shallow water system. J. Funct. Anal. 260 (2011), 1132-1154.

[38] Guan, C. And Yin, Z.: Global weak solutions for a modified two-component Camassa-Holm equation. Ann. Inst. H. Poincaré Anal. Non Linéaire 28 (2011), 623-641.

[39] Gui, G. And Liu, Y.: On the Cauchy problem for the two-component CamassaHolm system. Math. Z. 268 (2011), 45-66.

[40] Gui, G. And Liu, Y.: On the global existence and wave-breaking criteria for the two-component Camassa-Holm system. J. Funct. Anal. 258 (2010), 4251-4278.

[41] Henry, D.: Infinite propagation speed for the Degasperis-Procesi equation. J. Math. Anal. Appl. 311 (2005), 755-759.

[42] Holm, D., Marsden, J. and Ratiu, T.: The Euler-Poincaré equations and semidirect products with applications to continuum theories. Adv. Math. 137 (1998), 1-81.

[43] Holm, D., Ó NÁraigh, L. And Tronci, C.: Singular solution of a modified twocomponent Camassa-Holm equation. Phys. Rev. E (3) 79 (2009), 1-13.

[44] Holm, D. D. and Staley, M. F.: Wave structure and nonlinear balances in a family of evolutionary PDEs. SIAM J. Appl. Dyn. Syst. 2 (2003), 323-380 (electronic).

[45] Ionescu-Kruse, D.: Variational derivation of the Camassa-Holm shallow water equation. J. Nonlinear Math. Phys. 14 (2007), 303-312.

[46] Johnson, R.S.: Camassa-Holm, Korteweg-de Vries and related models for water waves. J. Fluid. Mech. 455 (2002), 63-82.

[47] Lenells, J.: Traveling wave solutions of the Degasperis-Procesi equation. J. Math. Anal. Appl. 306 (2005), 72-82.

[48] Liu, Y. And Yin, Z.: Global existence and blow-up phenomena for the DegasperisProcesi equation. Comm. Math. Phys. 267 (2006), 801-820.

[49] Lundmark, H.: Formation and dynamics of shock waves in the Degasperis-Procesi equation. J. Nonlinear Sci. 17 (2007), 169-198.

[50] Lundmark, H. And Szmigielski, J.: Multi-peakon solutions of the DegasperisProcesi equation. Inverse Problems 19 (2003), 1241-1245.

[51] Matsuno, Y.: Multisoliton solutions of the Degasperis-Procesi equation and their peakon limit. Inverse Problems 21 (2005), no. 5, 1553-1570.

[52] Marsden, J. AND Ratiu, T.: Introduction to mechanics and symmetry. A basic exposition of classical mechanics and systems. Second edition. Texts in Applied Mathematics 17, Springer-Verlag, New York, 1999.

[53] Popowicz, Z.: A 2-component or $N=2$ supersymmetric Camassa-Holm equation. Phys. Lett. A 354 (2006), 110-114.

[54] Popowicz, Z.: A two-component generalization of the Degasperis-Procesi equation. J. Phys. A 39 (2006), 13717-13726.

[55] Rodríguez-Blanco, G.: On the Cauchy problem for the Camassa-Holm equation. Nonlinear Anal. 46 (2001), no. 3, Ser. A: Theory Methods, 309-327.

[56] TAN, W. AND YIN, Z.: Global dissipative solutions of a modified two-component Camassa-Holm shallow water system. J. Math. Phys. 52 (2011), no. 3, 033507, 1-24.

[57] TAN, W. AND Yin, Z.: Global conservative solutions of a modified two-component Camassa-Holm shallow water system. J. Differential Equations 251 (2011), $3558-3582$. 
[58] Tan, W. And Yin, Z.: Global periodic conservative solutions of a periodic modified two-component Camassa-Holm equation. J. Funct. Anal. 261 (2011), 1204-1226.

[59] Toland, J. F.: Stokes waves. Topol. Methods Nonlinear Anal. 7 (1996), 1-48.

[60] Triebel, H.: Interpolation theory, function spaces, differential operators. NorthHolland Mathematical Library 18, North-Holland, Amsterdam, New York, 1978.

[61] Vakhnenko, V. O. and Parkes, E. J.: Periodic and solitary-wave solutions of the Degasperis-Procesi equation. Chaos Solitons Fractals 20 (2004), 1059-1073.

[62] Whitham, G. B.: Linear and nonlinear waves. Pure and Applied Mathematics, John Wiley \& Sons, New York-London-Sydney, 1974.

[63] Xin, Z. And Zhang, P.: On the weak solutions to a shallow water equation. Comm. Pure Appl. Math. 53 (2000), 1411-1433.

[64] YAn, K. AND Yin, Z.: Analytic solutions of the Cauchy problem for two-component shallow water systems. Math. Z. 269 (2011), 1113-1127.

[65] Yan, K. And Yin, Z.: On the Cauchy problem for a two-component DegasperisProcesi system. J. Differential Equations 252 (2012), 2131-2159.

[66] Yin, Z.: On the Cauchy problem for an integrable equation with peakon solutions. Illinois J. Math. 47 (2003), 649-666.

[67] Yin, Z.: Global solutions to a new integrable equation with peakons. Indiana Univ. Math. J. 53 (2004), 1189-1209.

[68] YiN, Z.: Global weak solutions for a new periodic integrable equation with peakon solutions. J. Funct. Anal. 212 (2004), 182-194.

Received November 23, 2011.

KaI YAN: Department of Mathematics, Sun Yat-sen University, 510275 Guangzhou, P. R. China.

E-mail: yankai419@163.com

Zhaoyang Yin: Department of Mathematics, Sun Yat-sen University, 510275 Guangzhou, P. R. China.

E-mail: mcsyzy@mail.sysu.edu.cn

This work was partially supported by NNSFC (No. 11271382 and No. 10971235), RFDP (No. 20120171110014), and the key project of Sun Yat-sen University (No. c1185). Yan was partially supported by the 2013 Excellent Doctoral Dissertation Cultivate program of Sun Yatsen University. 